\title{
ESTUDIO MICROMORFOLÓGICO EN SITIOS ALFAREROS URU DE LA CUENCA Del río Araguaia, Goiás, Brasil
}

\author{
Jordana Batista Barbosa a , Marisa Coutinho Afonso ${ }^{\mathrm{b}}$ y Julio Cezar Rubin de Rubin ${ }^{\mathrm{c}}$
}

\begin{abstract}
Resumen
Los estudios geoarqueológicos con análisis microscópicos han sido cruciales para la identificación de suelos modificados por la acción humana. La micromorfología en Brasil ha sido utilizada en sitios, concheros y sambaquis del sur y sudeste del país, en abrigos rocosos de la región de Lagoa Santa (Minas Gerais) y en sitios de "Terra Preta» (tierra negra) en Amazonas y Acre. Los llamados suelos antrópicos tienen un papel decisivo en los procesos de adición y movimiento de residuos orgánicos, minerales y prácticas de manejo del suelo. Para identificar e interpretar los diversos tipos de marcas $y$ actividades humanas en el suelo es importante comprender primero cómo ocurren estos cambios. En principio, las alteraciones en el suelo inducidas por humanos pueden ser causadas por procesos de adición de materiales al suelo y estos son identificados en la lectura micromorfológica de láminas delgadas. Los sitios arqueológicos de alfareros se encuentran ubicados en la cuenca del río Araguaia. Los asentamientos ceramistas en el estado de Goiás se estudiaron con el fin de identificar cuándo y cómo se establecieron estos grupos en la región central del país. Estos estudios mencionan la existencia de un suelo oscurecido en los sitios, que hasta ahora no habia sido estudiado a través de investigaciones micromorfológicas. La relación entre las características del bioma de Cerrado, con sus altos indices de acidez, y la ocupación humana en un área dada es importante para entender la tafonomía del suelo. El uso de la micromorfología demostró que incluso en entornos ácidos es posible observar alteraciones posteriores a la sedimentación, eventos microestratigráficos, fragmentos microscópicos de carbón, huesos, diversos minerales, como calcio, fósforo, compuestos orgánicos y microfragmentos cerámicos, todos relacionados a las actividades antrópicas de los sitios alfareros.
\end{abstract}

Palabras clave: micromorfología, suelos antrópicos, Cerrado, grupos ceramistas, geoarqueología.

\section{Abstract \\ MICROMORPHOLOGICAL STUDY IN «URU» CERAMIST SITES IN THE ARAGUAIA RIVER, GOIÁS, BRAZIL}

Geoarchaeological studies with microscopic analysis have been crucial for the verification of soils modified by human action. Micromorphology in Brazil has been used at sites, shell mounds and sambaquis in the south and southeast of the country, in rocky shelters in the Lagoa Santa region (Minas Gerais) and at "Terra Preta" sites in Amazonas and Acre states. So-called anthropic soils play a decisive role in the processes of addition and movement of organic, mineral residues and soil management practices. To identify and interpret the various types of human marks and activities on the ground, it is important to first understand how these changes occur. In principle, human-induced soil disturbances can be caused by processes of adding materials to the soil and these are identified in the micromorphological reading of thin sections. The archaeological sites of potters are located in the Araguaia river basin. Potters settlements in Goiás state were studied in order to identify when and how these groups were implemented in the central region of the country. These studies mention the existence of a darkened soil in the sites that had not been studied by micromorphological investigations until now. The relationship between the characteristics of the Cerrado biome, with its high acidity indexes, and human occupation in a given area is important to understand the taphonomy of the soil. The use of micromorphology demonstrated that even in acidic environments, it is possible to observe post-deposition alterations, microstratigraphic events, microscopic fragments of carbon, bones, various minerals, such as calcium, phosphorous, organic compounds, and ceramic microfragments, all related to anthropic activities from the potter sites.

Keywords: Micromorphology, Anthropic Soils, Cerrado, Potter Groups, Geoarcheology.

a https://orcid.org/0000-0002-0874-0877. bbarbosa.jordana@gmail.com

b https://orcid.org/0000-0002-4397-8856. MAE/USP - Museu de Arqueologia e Etnologia da Universidade de São Paulo/ CNPq. marisa.afonso@usp.br

c https://orcid.org/0000-0001-9789-2559. Pontifícia Universidade Católica de Goiás/ CNPq. rubin@pucgoias.edu.br 


\section{Introducción}

Brasil es el quinto país más grande del mundo (IBGE 2020) y se caracteriza por una gran diversidad de relieves y biomas. Esta investigación se desarrolló en la región del medio oeste de Brasil, estado de Goiás, que también abarca los estados de Mato Grosso y Mato Grosso do Sul (IBGE 2020) más precisamente en la cuenca hidrográfica del río Araguaia (Fig. 1).

De acuerdo con Morais et al. (1998: 27), el Planalto Central Brasileiro, por «su posición geográfica y sus características topomorfológicas es confirmado como un importante centro de paso, convergencia y dispersión de la ocupación humana precolombina, a lo largo del tiempo» [traducido del portugués].

Según los datos disponibles, la región fue ocupada por grupos indígenas que practicaban el cultivo de huertos para horticultura y agricultura, como complemento de la caza y pesca; la fabricación de utensilios de cerámica de arcilla (Andreatta 1975, 1982; Wüst 1983; Robrahn-González 1996; Sirico 2010; Barreto 2010 y Schmidt 2010).

Se han llevado a cabo numerosos trabajos sobre la posición de estos grupos alfareros en la configuración del estado de Goiás (Andreatta 1982; Wüst 1983; Robrahn-González 1996; Sirico 2010 y Barreto 2010). Estas investigaciones pretendían registrar los posibles patrones de asentamiento y espacios habitacionales (Andreatta 1982; Wüst 1983), zonas de confluencia para desplazamientos (Robrahn-González 1996) y organización espacial de las aldeas con morfologías circulares como espacios públicos (Barreto 2010).

Según Oliveira y Viana (1999-2000: 161) la ubicación de los sitios arqueológicos a cielo abierto, con perfiles poco estratificados y profundos, junto con la densidad del material arqueológico encontrado, principalmente cerámica, llevó a investigadores e investigadoras a presentar diferentes interpretaciones sobre la función de estos asentamientos caracterizados como grandes aldeas: 1) ceremonial (Simonsen 1983, 1984); 2) habitación residencial (Wüst 1983); 3) habitación semi-permanente (Schmitz y Barbosa 1985); 4) habitación (Schmitz 1986); 5) de función no residencial (Wüst 1990). Los vestigios arqueológicos de cerámica para estos sitios se clasificaron en dos tradiciones cerámicas principales: aratu y uru (Oliveira y Viana 1999-2000).

Los sitios alfareros uru representan las grandes aldeas del norte y oeste (Robrahn-González 1996) y fueron definidos en 1974 por Schmitz, Wüst, Barbosa y Basile Becker (Schmitz et al. 1982) como una tradición cerámica de grupos horticultores que cultivaban mandioca y maíz en la cuenca de los ríos Tocantins y Araguaia (Schmitz, Barbosa, Wüst 1976; Schmitz y Barbosa 1985). Estos grupos habrían ocupado la región entre 800 d.C. y 1720 d.C. (Wüst y Barreto 1999).

Los sitios de la tradición aratu se refieren a las grandes aldeas del este (Robrahn-González 1996). Entre los años 1983 y 2001 se obtuvo una gran cantidad de información sobre esta tradición fechada entre 171 d.C. y 1470 d.C. Esto se debe a los estudios exhaustivos de grandes aldeas con patrones circulares asociados a la fase Mossâmedes ${ }^{1}$ realizados por las arqueólogas Irmhild Wüst (1978, 1982), Margarida Andreatta (1982), Erika Robrahn-González (1996) y Cristiana Barreto (2010).

Lo que diferencia la tradición uru y al mismo tiempo la caracteriza, es la presencia de tostadores y cuencos relativamente poco profundos, con bases planas, paredes inflexionadas, ocasionalmente reforzadas y decoración incisa (Fig. 2) (Oliveira y Viana 1999-2000). Estos tostadores tienen diámetros de hasta 50 centímetros y están asociados al procesamiento de la mandioca (Viana et al. 2011). Las inclusiones utilizadas en la producción de pasta cerámica consisten en varios tipos de cariapé2 (Viana et al. 2011).

La expansión de los grupos asociados a la tradición uru habría ocurrido, según RobrahnGonzález (1996), desde los sitios de la fase Itapirapuã, ubicados en el valle del río Araguaia (Schmitz et al. 1982: 65). Las evidencias de contactos culturales en la cerámica uru se definieron con base en: 1) el uso cada vez mayor de cariapé como antiplástico en sustitución del mineral: 2) cambios en la forma de los recipientes; 3) aumento en el tamaño de los recipientes (Robrahn-González 1996; 

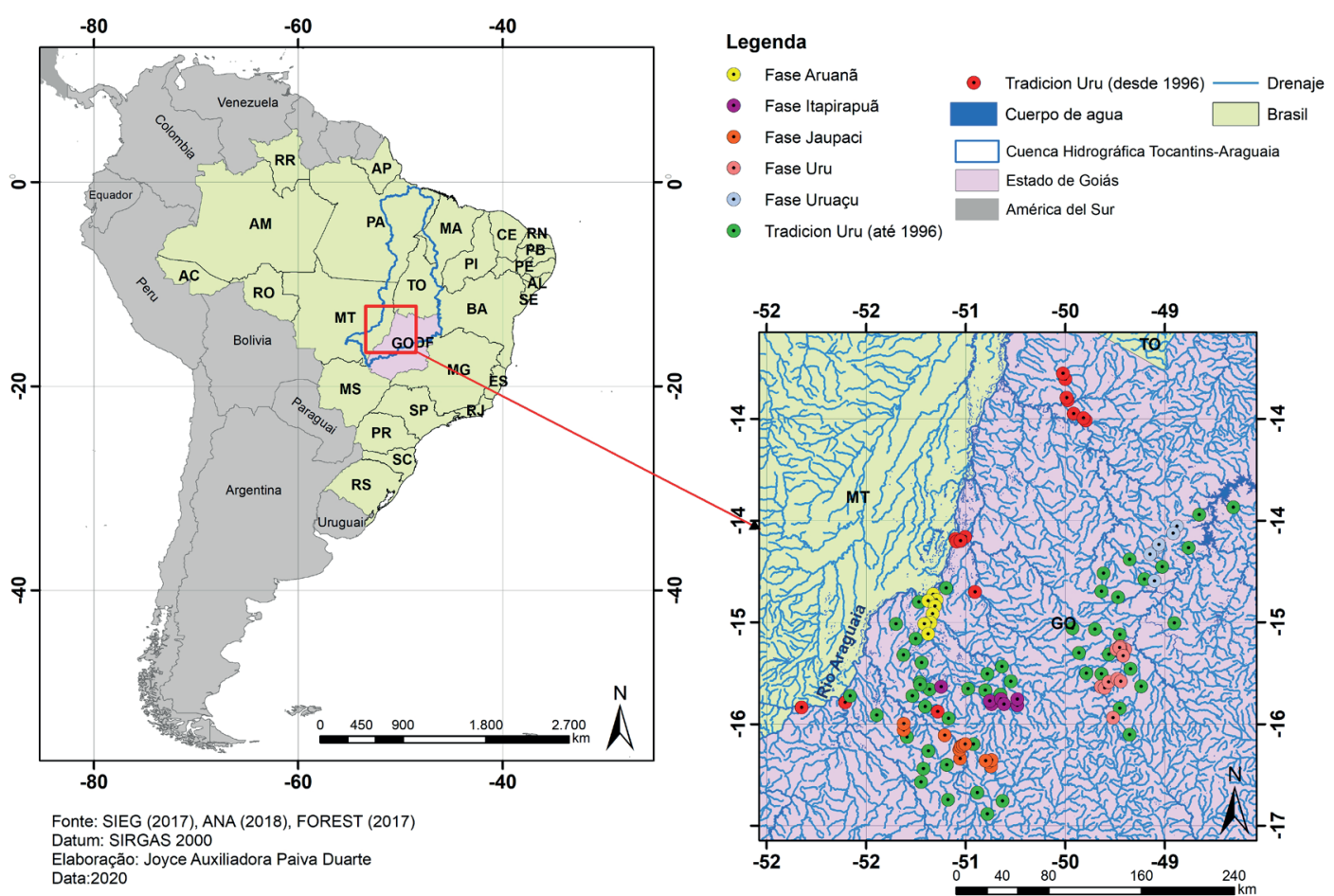

Tradicion Uru (até 1996)

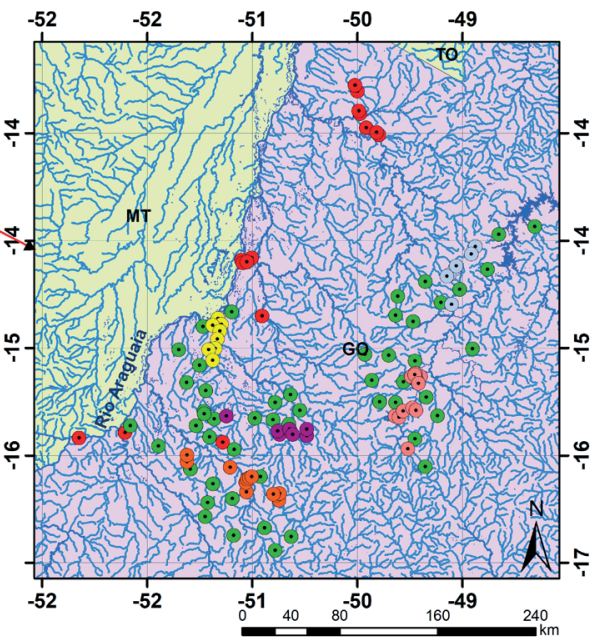

Figura 1. Ubicación del área de investigación con indicación de la cuenca hidrográfica. Ubicación de sitios de la tradición uru y fases arqueológicas (adaptado de Atlas Nacional do Brasil - Centro-Oeste; Schmitz et al. 1982; RobrahnGonzález 1996; SIEG 2017; Barbosa 2019).

Viana et al. 2011). Este proceso de cambio se identificó en las fases Uru de los sitios GO-Ni.28, fechado en $680 \pm 90$ años AP, y GO-Ni.35, fechado en $530 \pm 90$ años AP, y en la fase Jaupaci del sitio GO-Ju.47. Todos estos sitios presentaron evidencia del contacto de los grupos asociados a la tradición cerámica tupi guaraní (fase Iporá) con la tradición aratu, ubicados en la parte superior de Tocantins y medio Araguaia (Schmitz et al. 1982). Este contacto también sería responsable por el cambio en la alimentación, ya que pasa a predominar la mandioca, no el maíz, según lo que las autoras interpretan a partir de la forma de los contenedores (Robrahn-González 1996; Viana et al. 2011).

La reanudación de las investigaciones en los sitios Cangas I y Lago Rico tuvieron como objetivo identificar suelos antrópicos en los sitios alfareros en la cuenca del río Araguaia, utilizando métodos de geoquímica y micromorfología de depósitos arqueológicos. El uso de estas técnicas permitió confirmar la presencia de suelos orgánicos en sitios cerámicos en el estado de Goiás, además de probar la hipótesis establecida en investigaciones previas.

\section{Suelos antrópicos en sitios alfareros}

El suelo registra, en muchas situaciones, el paso de una población y la evidencia de las actividades realizadas por el grupo. Los suelos son una fuente potencial de información en estudios arqueológicos (Holliday 2004). Estos suelos impactados por la acción humana pueden subdividirse en: suelos antropogénicos, que se consideran alterados intencionalmente; y suelos antrópicos, o antrosoles, que han sido alterados sin intención humana (Eidt 1985: 23). 

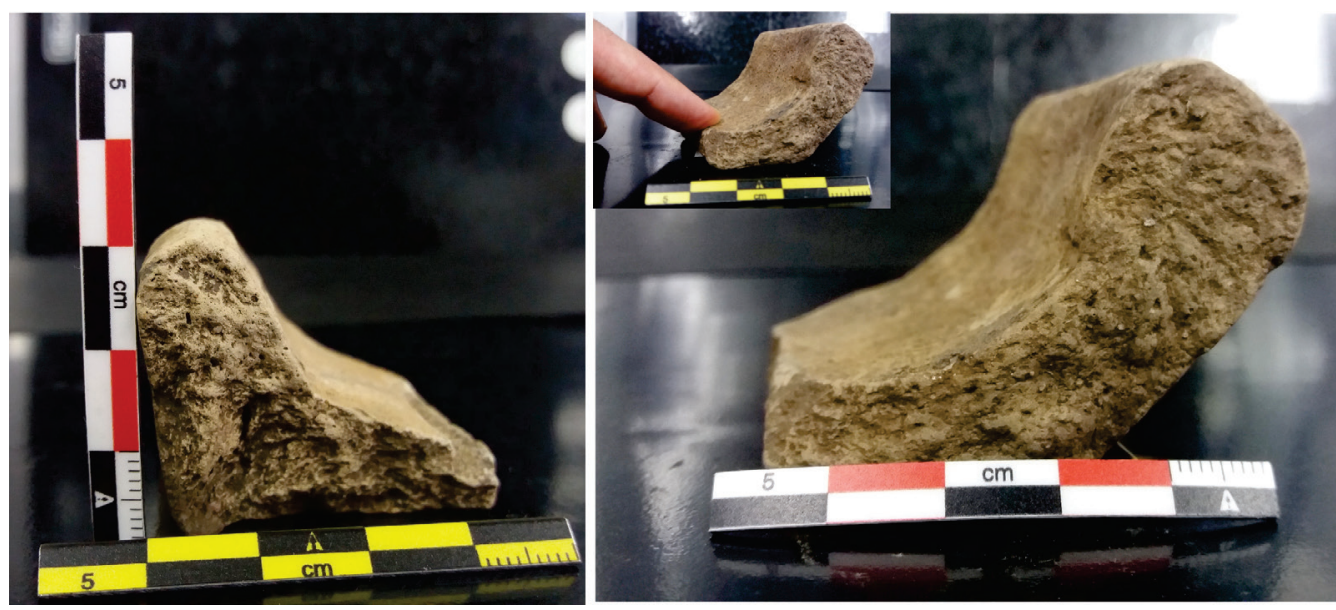

Figura 2. Cerámicas, base y borde (n³59 y 04) del sitio Lago Rico (fuente: Barbosa, 2019).

Los antrosoles se definen por horizontes diagnósticos que pueden resultar de la adición de material (IUSS Grupo de Trabajo WRB 2007: 71). La terminología y características de estos horizontes diagnósticos está basada en la referencia del Grupo de Trabajo World Reference Base (WRB, por sus siglas en inglés) (IUSS Grupo de Trabajo WRB 2007). De acuerdo con el WRB (2007) hay seis tipos de antrosoles: (1) hótico, (2) plágico, (3) térrico, (4) prético, (5) irrágico e (6) hidrágrico (Howard 2017: 118).

Los antrosoles se definen por horizontes diagnósticos que pueden resultar de la adición de material (IUSS Grupo de Trabajo WRB 2007WRB 2006: 71). La terminología y características de estos horizontes diagnósticos está basada en la referencia del Group in the World Reference Base (WRB, por sus siglas en inglés) (IUSS Grupo de Trabajo WRB 20076). De acuerdo con el WRB (20076) hay seis tipos de antrosoles: (1) hótico, (2) plágico, (3) térrico, (4) prético, (5) irrágico e (6) hidrágrico (Howard 2017: 118). Los suelos antropogénicos han sido influenciados, modificados o creados por la actividad humana, en contraste con los suelos formados por procesos naturales (Howard 2017). Términos como antrosol, suelo antrópico o antropogénico, implican la existencia de un perfil de suelo previamente desarrollado (Villagrán 2008: 22).

De acuerdo con el Sistema Brasileño de Clasificación de Suelos de la Embrapa (Santos et al. 2018) y Bennema (2006[1966]), el suelo antrópico es un horizonte formado o modificado por la actividad humana continua (Silva y Rebellato 2004; Kern et al. 2008; Costa et al. 2009; ArroyoKalin 2014; Teixeira y Lima 2016; Schmidt 2010), ya sea como lugar de residencia, de disposición o de cultivo, en el que haya evidencias de adición de material orgánico de naturaleza variable, mezclado o no con material mineral, que pueda comprobarse por la presencia de artefactos cerámicos y/o líticos, huesos, conchas o rastros de acción de fuego, como hogares, dejando vestigios de carbón y cenizas.

Según Schmidt (2010), los suelos antrópicos identificados en los sitios alfareros revelan la organización interna de la experiencia de las personas en el lugar habitado, así como la morfología y los registros de eventos económicos, sociales e históricos.

\subsection{Micromorfología y química multielemental}

Los cambios en el suelo inducidos por los humanos pueden ser causados por procesos morfológicos, físicos, químicos, mineralógicos e incluso magnéticos (Eidt 1985; Teixeira y Lima 2016; Macphail et al. 1990). Estos cambios pueden reflejar la naturaleza de las actividades humanas, a través de la identificación de los procesos de depositación y posdepositación (sensu Schiffer 1972). 
La micromorfología estudia los constituyentes de los suelos y sedimentos y sus relaciones, con el objetivo de identificar los procesos responsables de su formación y transformación, así como para explicar algunas de sus características particulares (Villagran 2008). Esta técnica ha ayudado a numerosas investigaciones (Araujo et al. 2008; Villagran et al. 2009; Villagran et al. 2010; Villagran et al. 2011; Feathers et al. 2010; Villagran 2014; Villagran y Giannini 2014; Villagran et al. 2017; Lahaye et al. 2019) a identificar eventos microestratigráficos, a través del estudio de los procesos de formación del sitio, aportando al análisis físico y químico. Los estudios micromorfológicos desempeñan un papel cada vez más destacado en las investigaciones geoarqueológicas, desde trabajos sobre génesis del suelo y tafonomía, hasta el establecimiento de la naturaleza, el origen y la importancia de los depósitos geogénicos y antrópicos y sus entornos asociados (Courty et al. 1989; Macphail y Cruise 2001; Ruivo et al. 2004: Villagran 2018).

Los sitios estudiados en la Amazonía son buenos ejemplos del uso de análisis químicos para analizar antrosoles en sitios alfareros (Heckenberger 2005; Schmidt 2010). En estos contextos, los cambios antrópicos en el paisaje y en el suelo, se han convertido en piezas clave para las discusiones sobre los antiguos pueblos alfareros (Wüst 1983; Heckenberger 2005; Schmidt y Heckenberger 2009; Schmidt 2010; Barbosa 2019).

Observaciones etnográficas sugieren que casas, vertederos y jardines serían contextos ideales para generar niveles altos de $\mathrm{C}, \mathrm{Ba}, \mathrm{Ca}, \mathrm{Co}, \mathrm{Cr}, \mathrm{Cu}, \mathrm{Fe}, \mathrm{K}, \mathrm{Mg}, \mathrm{Mn}, \mathrm{Na}, \mathrm{Ni}, \mathrm{P}, \mathrm{Pb}, \mathrm{Sr}, \mathrm{Ti}, \mathrm{V}$ y Zn, los cuales documentan la formación de suelos ricos en materia orgánica a partir de la gestión de residuos, acumulación de fertilizantes orgánicos y quema de coivara (Zeidler 1983; Posey 1984; Heckenberger et al. 1999; Erickson 2003; Hecht 2003; Denevan 2004; Silva y Rebellato 2004; Heckenberger 2005; Arroyo-Kalin 2008; Schmidt y Heckenberger 2009; Schmidt 2010).

\section{3. Área de estudio}

Los sitios arqueológicos Cangas I, ubicado en la terraza aluvial del río Araguaia, y el sitio Lago Rico, ubicado en la pendiente a lo largo de la margen izquierda del río do Peixe, fueron estudiados a lo largo de cinco años, produciendo numerosas publicaciones sobre la ocupación precolonial de la región (Rosa 2009; Pereira 2010; Marcos 2011; Correa 2013; Silva 2013; Moura 2015; Barbosa 2015; Ortega 2016; Estrela 2017; Rubin et al. 2019).

Durante la investigación realizada en el sitio Cangas I se abrieron dos unidades de excavación que permitieron realizar la caracterización estratigráfica del sitio e identificar el área de ocupación. Se evidenciaron tres capas arqueoestratigráficas en el sitio Cangas I con las siguientes características: A) grosor promedio de 50 centímetros, contiene artefactos cerámicos y líticos y residuos contemporáneos, así como estructuras de combustión (Pereira 2010: 2) identificadas como sedimentos de color gris claro (2.5YR 5/0), arcilla limosa y presencia de clastos pedogenizados; Ab) estéril en términos arqueológicos, tiene un espesor promedio de 50 centímetros, color gris claro (2.5 YR $7 / 0$ ), textura de arena fina y poca bioturbación; B) correspondiente a la formación Araguaia ${ }^{3}$, tiene color amarillo (10 YR 7/6), espesor promedio de 230 centímetros y textura de arena fina, sin estructuras sedimentarias (Marcos 2011). Entre 20 y 40 centímetros de profundidad, durante la excavación de la Unidad 1, se identificaron estructuras de combustión y marcas de poste. Se registraron fragmentos de huesos entre los 10 y 20 centímetros y entre los 80 y 90 centímetros (Fig. 3) (Rosa 2009).

Seis fechados fueron producidos para el sitio Cangas I (Tabla 1). Todas las muestras fueron tomadas de la Unidad de excavación 2, la cual presentó un contexto mejor preservado y menos afectado (Rubin 2007; Rosa 2009). De esta manera, se obtuvieron tres fechados radiocarbónicos sobre la base de muestras de carbón, y tres fechados por luminiscencia ópticamente estimulada (LOE) a partir de fragmentos cerámicos (Fig. 4). 

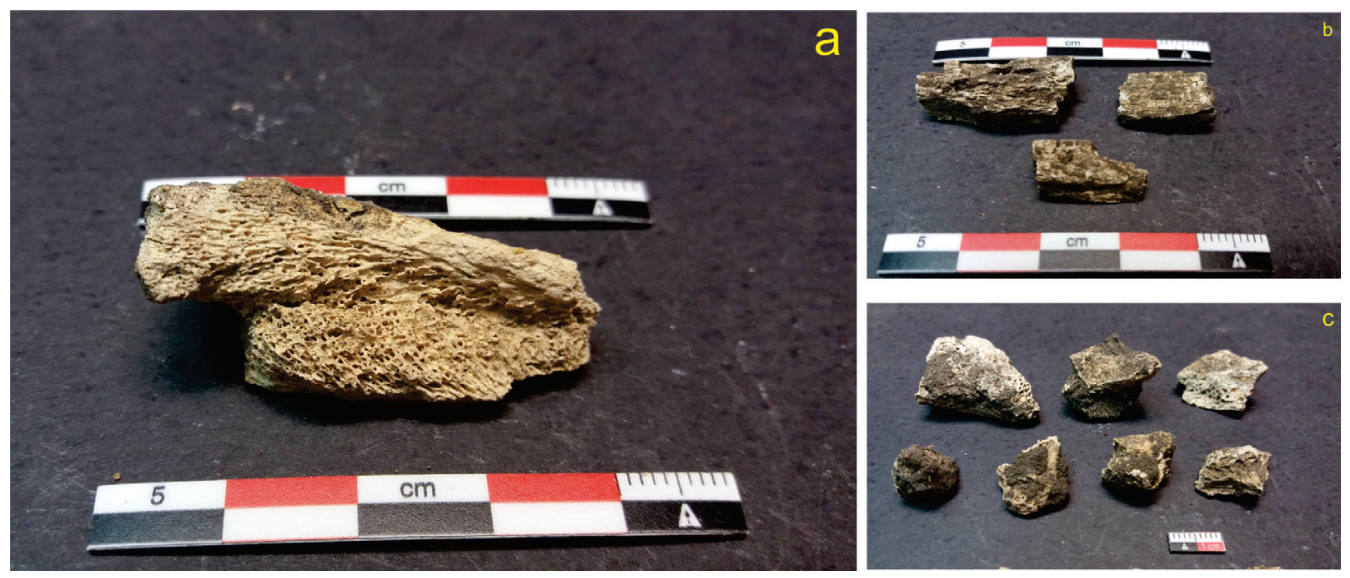

Figura 3. Fragmentos de hueso encontrados durante la excavación de la Unidad 1 del sitio Cangas I: a) cuadrícula $1 \mathrm{~A}$ de $80-90 \mathrm{~cm}$; b) cuadricula $1 B$ de $10-20 \mathrm{~cm}$; c) cuadricula $1 \mathrm{~A}$ de $80-90 \mathrm{~cm}$.

Tabla 1. Fechas obtenidas por los métodos de radiocarbono y luminiscencia ópticamente estimulada (LOE) para el sitio Cangas I, Unidad de Excavación - 2. Los acrónimos $3 E, 1 A, 1 B, 2 A, 2 B$ y $1 F$ se refieren al cuadrante de donde se obtuvieron muestras para datación (fuente: Rubin et al. 2019).

\begin{tabular}{|c|c|c|c|c|c|c|}
\hline $\mathrm{N}^{\circ}$ & Muestra & $\begin{array}{l}\text { Unidad de } \\
\text { excavación }\end{array}$ & Profundidad & $\begin{array}{l}\text { Método } \\
\text { utilizado }\end{array}$ & $\begin{array}{l}\text { Material } \\
\text { fechado }\end{array}$ & $\begin{array}{l}\text { Cronología } \\
\text { obtenida }\end{array}$ \\
\hline 1 & $\begin{array}{l}\text { Beta } 452046 \\
\text { (CG-C01) }\end{array}$ & $\mathrm{UE}-2$ / 3E & $50 \mathrm{~cm}$ & Radiocarbónico & $\begin{array}{l}\text { Carbón asociado a } \\
\text { cerámica }\end{array}$ & $\begin{array}{c}570+/-30 \\
\text { años AP }\end{array}$ \\
\hline 2 & $\begin{array}{l}\text { Beta } 452047 \\
(\text { CG-C02) }\end{array}$ & $\begin{array}{l}\mathrm{UE}-2 / 1 \mathrm{~A}, 1 \mathrm{~B} \\
2 \mathrm{~A} \text { e } 2 \mathrm{~B} .\end{array}$ & $60 \mathrm{~cm}$ & Radiocarbónico & $\begin{array}{l}\text { Carbón (estructura } \\
\text { de combustión) }\end{array}$ & $\begin{array}{c}610+/-30 \\
\text { ańos } \mathrm{AP}\end{array}$ \\
\hline 3 & $\begin{array}{l}\text { Beta } 452048 \\
(\mathrm{CG}-\mathrm{C} 0)\end{array}$ & UE-2 / 1F & $50 \mathrm{~cm}$ & Radiocarbónico & $\begin{array}{l}\text { Carbón asociado a } \\
\text { cerámica }\end{array}$ & $\begin{array}{c}460+/-30 \\
\text { años AP }\end{array}$ \\
\hline 4 & Datación 4540 & $\mathrm{UE}-2 / 1 \mathrm{~F}$ & $50 \mathrm{~cm}$ & LOE & Cerámica & $244+/-40$ \\
\hline 5 & Datación 4541 & UE-2 / 3E & $50 \mathrm{~cm}$ & LOE & Cerámica & $234+/-30$ \\
\hline 6 & Datación 4542 & $\begin{array}{l}\mathrm{UE}-2 \text { / } 1 \mathrm{~A}, 1 \mathrm{~B} \\
2 \mathrm{~A} \text { e } 2 \mathrm{~B}\end{array}$ & $50 \mathrm{~cm}$ & LOE & Cerámica & $284+/-40$ \\
\hline
\end{tabular}

Las fechas obtenidas para el sitio Cangas I muestran variaciones de tiempo a intervalos de 10 centímetros, o, como en las cuadrículas $3 \mathrm{E}$ y $1 \mathrm{~F}$, en el mismo nivel, con una diferencia de 100 años. Esto puede sugerir dos o más momentos de ocupación (Rubin et al. 2019).

En el sitio Lago Rico se excavaron tres unidades con 25 cuadrículas de $1 \mathrm{~m}^{2}$. Durante las excavaciones, se utilizaron cuatro perfiles estratigráficos como referencia para la caracterización de depósitos y el análisis físico-químico. Se evidenciaron tres capas arqueoestratigráficas en todo el sitio: A) espesor medio de 30 centímetros, mal estructurado, textura entre arenosa y arenosaarcillosa, baja pegajosidad, predominio de granos angulares de cuarzo y coloración variegada entre marrón oscuro (7.5 YR 4/2) seco y marrón grisáceo (10 YR 3/2) húmedo con mayor porcentaje de material arqueológico; $\mathrm{Ab}$ ) tiene un espesor promedio de 15 a 25 centímetros, estructura pobre, textura entre arena arenosa y arcillosa con baja pegajosidad, predominio de granos angulares de cuarzo (Wentworth 1922), coloración variegada entre marrón oscuro (7.5 YR 4/4) y marrón oscuro (7.5YR 3/2) húmedo; B) tiene un color predominantemente amarillo claro (2.5 YR 7/4, 


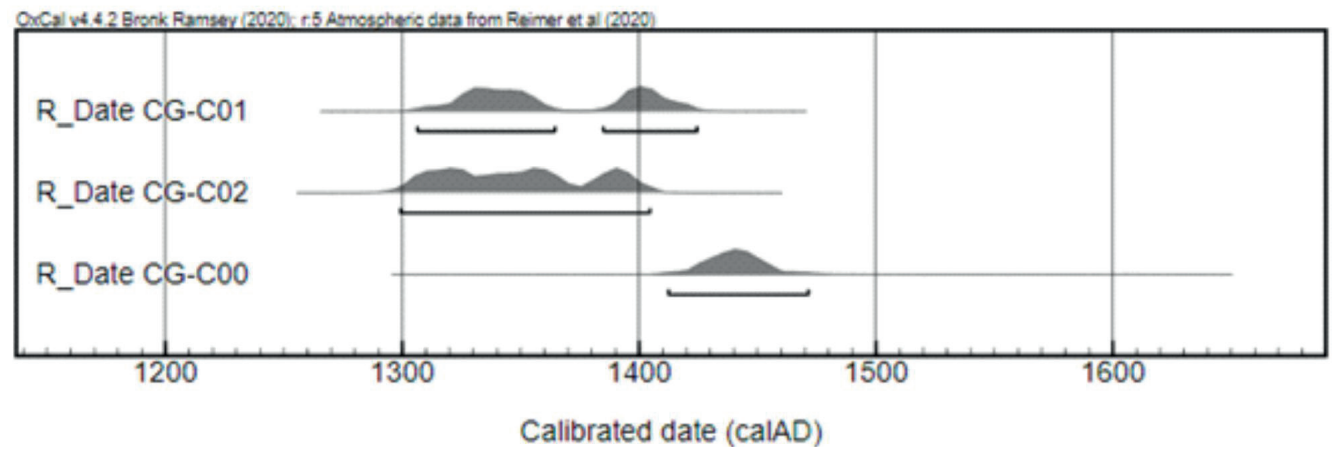

Figura 4. Fechas obtenidas por métodos de radiocarbono calibrado 2 sigmas (95\%) en OxCal 4.4, curva de calibración ShCal 13 (fuente: Hogg et al. 2013; Bronk Ramsey 2017).

contiene algunos fragmentos (arena muy gruesa y gránulos) de gneis, que pueden estar relacionados con la roca madre, según el mapa geológico de Moreira et al. (2008).

Para el sitio Lago Rico se realizaron análisis químicos que revelaron valores relativamente altos en relación con los valores naturales encontrados en los suelos del Cerrado (Barbosa 2015 y Barbosa 2019). Los resultados llevaron a dos hipótesis para explicar el origen de las propiedades químicas: 1) actividades agrícolas y ganaderas recientes; 2) actividades humanas precoloniales, ya que se encontraron grandes cantidades de fragmentos de cerámica dispersos en un área de $360,000 \mathrm{~m}^{2}$. Para el sitio Lago Rico, se obtuvieron tres fechas por LOE sobre la base de fragmentos de cerámica, debido a que no se encontró ningún carbón en el contexto arqueológico (Tabla 2).

Tabla 2. Fechas obtenidas por luminiscencia ópticamente estimulada (LOE) para la UE-2 del sitio Lago Rico (fuente: Rubin et al. 2019).

\begin{tabular}{ccccccc}
\hline$N^{\circ}$ & Muestra & $\begin{array}{c}\text { Unidad de excavación - } \\
\text { cuadrícula }\end{array}$ & Profundidad & $\begin{array}{c}\text { Método } \\
\text { utilizado }\end{array}$ & $\begin{array}{c}\text { Material } \\
\text { fechado }\end{array}$ & $\begin{array}{c}\text { Cronología } \\
\text { obtenida }\end{array}$ \\
\hline 1 & Datación 4543 & UE-2/3C & $30 \mathrm{~cm}$ & LOE & Cerámica & 254+/-35 \\
\hline 2 & Datación 4544 & UE-2/2D & $30 \mathrm{~cm}$ & LOE & Cerámica & $484+/-75$ \\
\hline 3 & Datación 4545 & UE-2/3D & $35 \mathrm{~cm}$ & LOE & Cerámica & $534+/-35$ \\
\hline
\end{tabular}

Los análisis de la cultura material de los sitios de Lago Rico (Ortega 2016; Estrela 2017) y Cangas I (Melo 2009) indicaron que las morfologías de los recipientes de cerámica son similares a las de la tradición Uru, con recipientes predominantemente sin decoración, bases planas, formas de platos y pequeñas angulaciones o muescas en los labios, bases en pedestal y bordes presentando decoración solo en algunas pocas piezas (Melo 2009; Ortega 2016; Estrela 2017).

Después de diez años de investigación, se ha establecido la hipótesis de que el sitio Cangas I podría ser un sitio temporal y el Lago Rico una aldea principal (Marcos 2011). Otros trabajos desarrollados en el sitio Lago Rico posteriormente, llegaron a conclusiones similares. Según las investigaciones, este sitio presenta características análogas a un sitio aldea, considerando las dimensiones espaciales y el contexto ambiental (Correa 2013; Silva 2013; Moura 2015; Barbosa 2015). 


\section{Nuevas perspectivas}

\subsection{Métodos}

El estudio geoarqueológico de los sitios Cangas I y Lago Rico estuvo dirigido a realizar nuevos muestreos de suelo para análisis físicos, químicos, mineralógicos y micromorfológicos. La descripción en campo incluyó la identificación y descripción de estructuras sedimentarias y arqueológicas, así como aspectos sedimentológicos (Goldberg y Macphail 2006). El método de recolección del suelo se eligió con base en datos primarios, que encontraron que los sitios arqueológicos están hasta 50 centímetros de profundidad (Barbosa 2015).

Las muestras para micromorfología se llevaron a cabo con el objetivo de responder a la siguiente pregunta: ¿las concentraciones de elementos químicos identificados en los sitios Lago Rico son el resultado de procesos naturales, agrícolas modernos o humanos precoloniales? ¿Cuál es el origen de la matriz oscura identificada en el sitio Cangas I y Lago Rico? ¿Cuál es el origen de los altos valores de fósforo presentes en el sitio Cangas I? Para eso, fueron tomadas muestras dentro del área delimitada como sitio y fuera del área, para obtener datos de referencia que auxilien a interpretar el contexto general de los sitios (Figs. 5 y 6).

\subsection{Muestreo}

Dentro del área del sitio Lago Rico se abrió una trinchera cerca a la unidad de excavación donde los valores de los elementos químicos, según Barbosa (2015), se pueden comparar con los suelos antrópicos. La ubicación de la cuadrícula fuera del área definida como sitio, fue en base a la ausencia de rastros culturales superficiales (Barbosa 2015). Para el sitio Cangas I, el muestreo se realizó cerca de la unidad de excavación donde se obtuvieron las fechas para el sitio.

Al preparar el perfil para comenzar el muestreo para micromorfología, determinamos que las colecciones seguirían las capas ya establecidas en investigaciones previas: capas 1 y 2, perfil este para el sitio Lago Rico y perfil norte para el sitio Cangas I. Se utilizaron muestras de cajas de papel previamente ensambladas, colocadas en el perfil en una ubicación previamente marcada. Sin quitar la caja del lugar y usando una espátula y una cuchara de jardinería, se esculpieron los lados de la caja (Figs. 7a y 7b), permitiendo que estos lados se profundicen gradualmente en el perfil. Después del muestreo, los bloques se empacaron en una película de plástico y se etiquetaron con la información de campo y la orientación en la que se tomaron las muestras, para evitar errores al enviarlas al laboratorio (Figs. 7c y 7d). En el laboratorio, las muestras fueron secadas, impregnadas y laminadas para observación en microscopio.

Las descripciones de las secciones delgadas se realizaron con microscopio Leica 2700P bajo luz polarizada plana (PPL) y luz polarizada cruzada (XPL), en aumentos de $2.5 x$ hasta $50 x$ siguiendo la guía de Stoops (2003) y Bullock (1985). Al mismo tiempo, se realizaron fotomicrografías utilizando el software LAS v.10.

\section{Resultados}

Las muestras del sitio Lago Rico se recolectaron en el horizonte A, entre 0 y 20 centímetros, y en el horizonte B, entre 20 y 40 centímetros. La recolección LR-01 corresponde al horizonte B, que destaca por su coloración 2.5 YR 7/4 (seca), más clara y con textura más arcillosa. Con el análisis microscópico se confirmó que este horizonte se refiere al horizonte $\mathrm{B}$, debido a la presencia de revestimientos de arcilla clara y laminar característicos (Figs. 8A y 8B).

La relación gruesos/finos ${ }^{4}$ (g/f) de la muestra LR-01 es de 60/40. La fracción fina que conforma la sección delgada tiene coloración amarillo-anaranjado y presencia de puntos rojos (nódulos de óxido de hierro). La fracción gruesa contiene granos de cuarzo subangulares, pobremente 


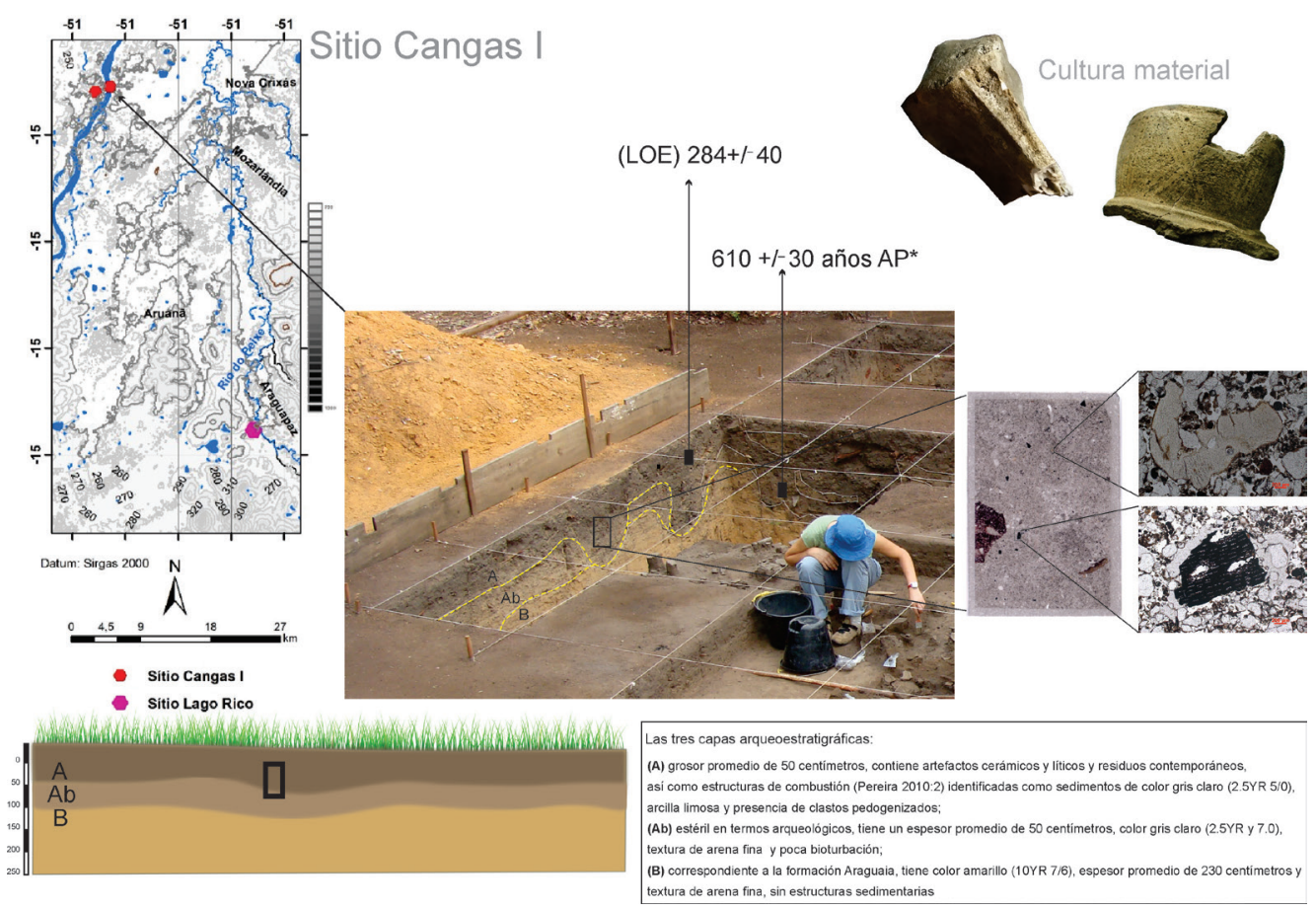

Figura 5. Croquis del área de muestreo - Sitio Cangas I.

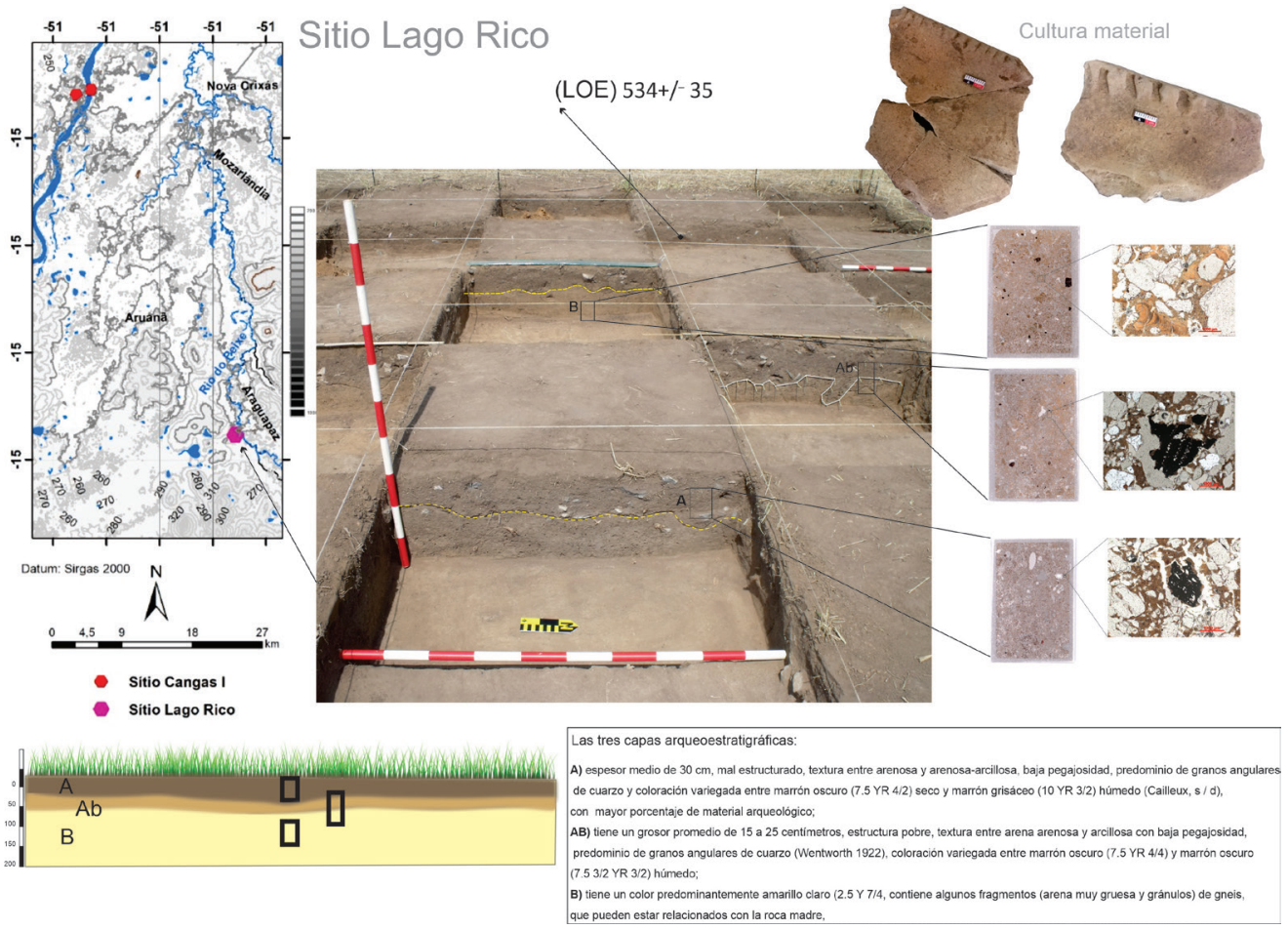

Figura 6. Croquis del área de muestreo - Sitio Lago Rico 

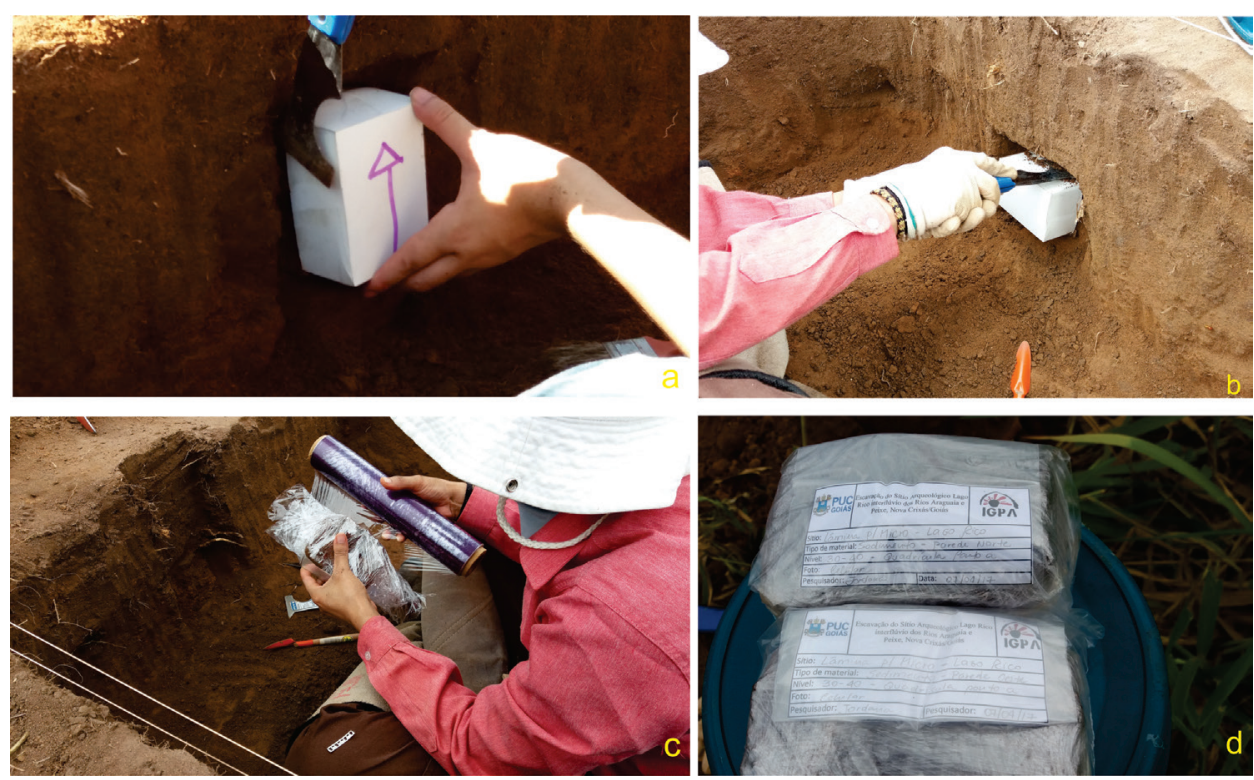

Figura 7. Procedimiento de muestreo. a) posicionamiento de la caja en el área muestreada; b) excavación de los lados de la muestra; c) acondicionamiento de la muestra en una película de plástico; d) muestras acondicionadas y etiquetadas.

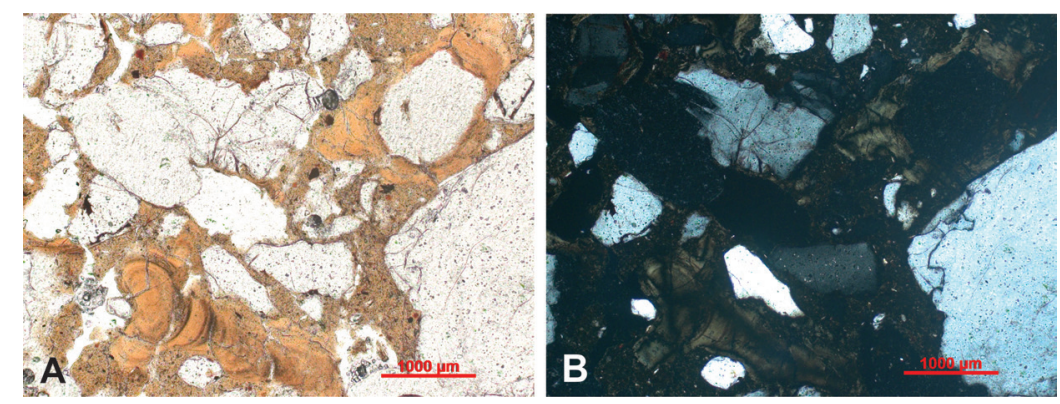

Figura 8. Fotomicrografias de la sección delgada LR-01: A) recubrimientos de arcilla con 20 aumentos, en PPL; B) revestimientos de arcilla con 20 aumentos, en XPL (fuente: Barbosa 2019).

seleccionados, y nódulos de hierro redondeados en la fracción arena muy gruesa, con distribución aleatoria. El análisis micromorfológico mostró porosidad cubriendo aproximadamente el 35\% del área de la sección delgada.

Alrededor del 20\% corresponde a poros compuestos por cámaras (Fig. 9A) y 15\% del tipo empaquetamiento complejo, con distribución aleatoria. El ancho de los poros en cámara está entre $100 \mu \mathrm{m}$ y $200 \mu \mathrm{m}$. En la fracción gruesa, también están presentes las raíces en forma fibrosa (Fig. 9B), con aproximadamente 3\% de fragmentos de carbón (Fig. 9C y 9D), también hay presencia de excrementos globulares agrupados (Fig. 9E y 9F). Los rasgos edáficos incluyen revestimientos de arcilla laminares, nódulos y excrementos.

La muestra LR-02 corresponde al horizonte A (antrópico) y se destaca por la abundancia de fragmentos cerámicos. Este horizonte tiene un color de 7.5 YR 4/2 (seco) y 10 YR 3/2 (húmedo). La porosidad cubre aproximadamente el $20 \%$ del área de la lámina. Alrededor del $5 \%$ corresponde a poros compuestos por cámaras entre $100 \mu \mathrm{m}$ y $200 \mu \mathrm{m}$. Poros de empaquetamiento complejo 

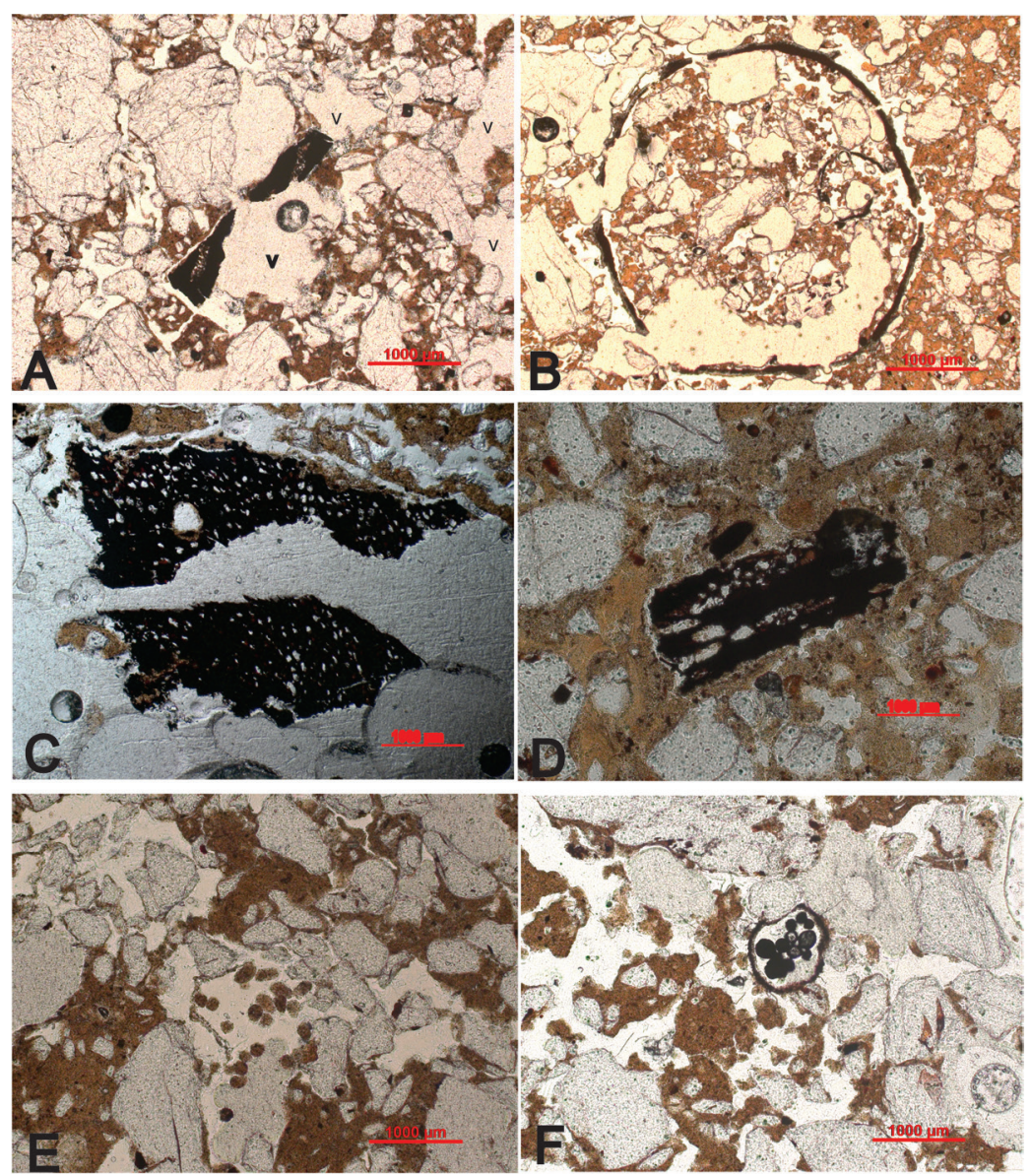

Figura 9. Fotomicrografias de la sección delgada LR-01: A) Distribución relacionada con la fracción gefúrica g/f, cóncava y vacía $(V)$, tipo de cámara, aumento de $5 x$, en PPL; B) Resto de tejido orgánico (raíces) en PPL, con un aumento de 2.5x; C) Carbón, aumento de 20x en PPL; D) Carbón, aumento de 10x en PPL; E) Excrementos, aumento de 10x, en PPL; F) Excremento en forma globular, aumento de 10x, en PPL (fuente: Barbosa 2019).

están presentes en el otro 15\%, y la distribución se considera aleatoria. La relación g/f es de 30/70. La fracción gruesa contiene granos de cuarzo subangulares, pobremente seleccionados; nódulos redondeados de hierro y manganeso en la fracción arena muy gruesa y arena muy gruesa, con distribución y orientación aleatoria.

La fracción fina muestra dos matrices: color amarillo/naranja, básicamente compuesto de limo y arcilla, transparente y laminar en PPL, con la presencia de pequeńos puntos rojos, y fabrica-b, que es indiferenciada en XPL. En la fracción gruesa, las raíces están presentes en forma fibrosa, con una abundancia de aproximadamente $4 \%$ clasificadas con distribución y orientación aleatoria. En las raíces, se identificaron excrementos de ácaros elipsoides dentro o al lado de ellos.

Incluso en la fracción gruesa están presentes los tipos de nódulos que se clasifican según el tipo de estructura (Stoops 2003). Los nódulos de óxido de hierro identificados en esta sección tienen una característica anórtica y se forman a través de agregados (Figs. 10A y 10B). Los fragmentos de carbón con esfericidad ondulante, presentes en 3\%, se distribuyen aleatoriamente. Para este horizonte, el carbón aparece con un porcentaje ligeramente superior al del horizonte $\mathrm{B}$, lo que puede justificar la presencia de nutrientes con una mayor concentración (Fig. 10C). Como se puede ver 


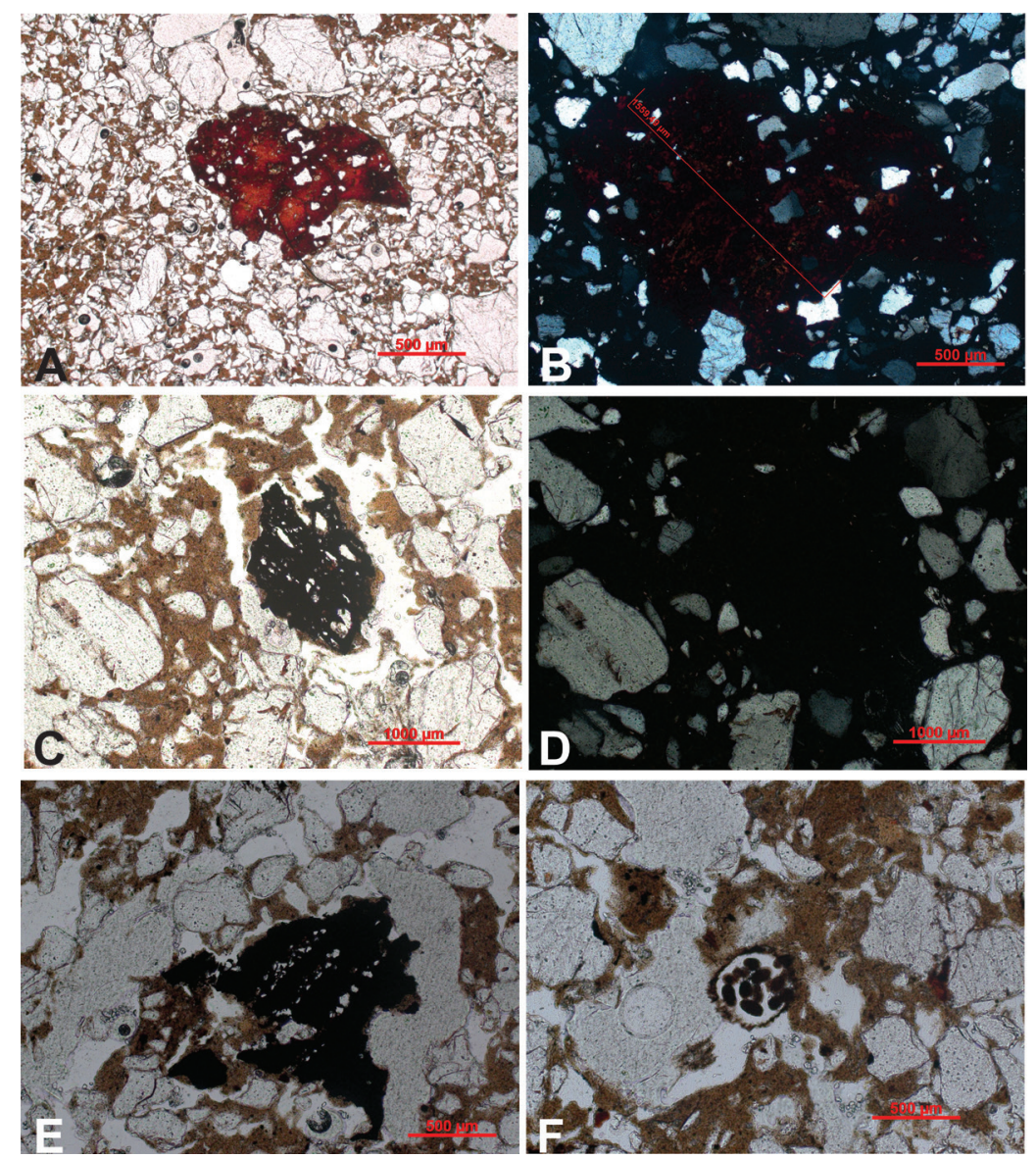

Figura 10. Fotomicrografias del portaobjetos LR-02: A) Nódulo órtico, con aumento de 2.5x, en PPL; B) Nódulo anórtico con 5 aumentos, en XPL, y longitud de $1559 \mu \mathrm{m}$; C) Fragmento de carbón, con aumento de 10x, en PPL; D) Fragmento de carbón, con aumento de 10x, en XPL; E) Fragmento de carbón, con aumento de 10x, en PPL; F) Excremento - Mitoides, con un aumento de 10x, en PPL (fuente: Barbosa 2019).

(Fig. 10D), el carbón no tiene birrefringencia en XPL, y en la figura 10E es posible ver claramente las células del fragmento de carbón con revestimientos de arcilla.

En la fracción gruesa también tenemos excrementos, presentes en solo 1\%, con forma globular y distribución en grupos. Estos excrementos tienen una distribución y orientación aleatoria, clasificada como Mitoids, con coloración roja/amarilla y composición orgánica. Este tipo de excremento tiene una sección longitudinal alargada y, en la sección transversal, pequeños círculos (Fig. 10F). Las características pedológicas identificadas en esta sección delgada, incluyen recubrimientos de arcilla paralelos y laminares, así como nódulos de hierro y excrementos de ácaros.

La recolección LR-03 corresponde a la transición del horizonte A al horizonte B. Elegimos realizar la recolección de micromorfología entre las capas para descubrir si estos horizontes están realmente fuera de lo que se consideró en la delimitación del sitio arqueológico. La coloración de estas capas es similar a las otras dentro del área del sitio, siendo 2.5 YR 7/2 (seco) más claro y con una textura de suelo más arcillosa. En esta cuadrícula, no se encontraron rastros de cerámica en la superficie y en el subsuelo, que se consideran estériles.

Con el análisis microscópico utilizando aumentos de $2.5 \mathrm{x}$ a $50 \mathrm{x}$, se identificaron las siguientes características: porosidad, que cubre alrededor del 25\% del área en la sección delgada: 


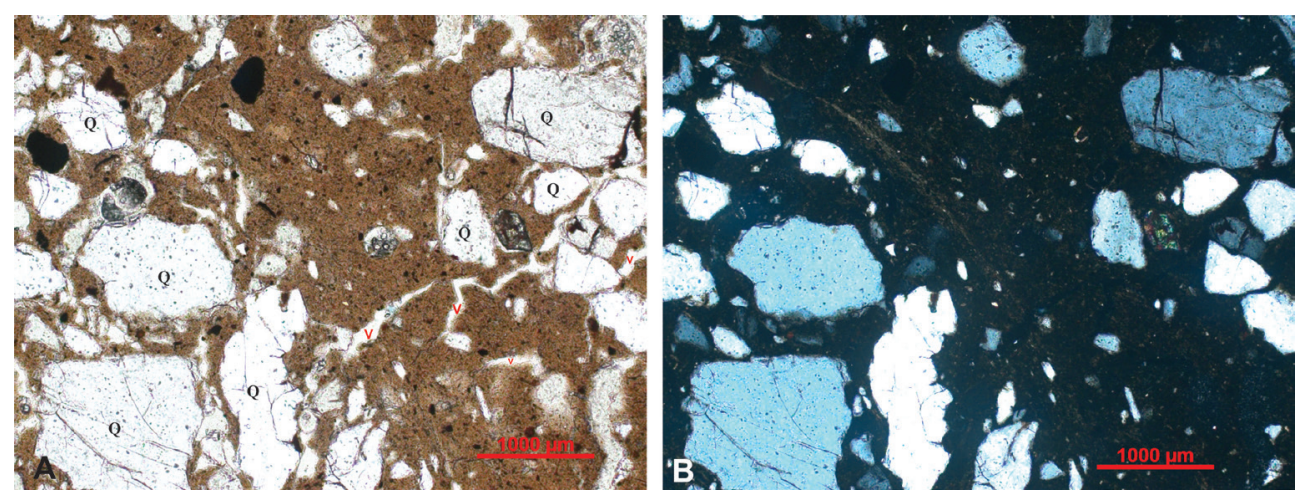

Figura 11. Fotomicrografías de la lámina LR-03: A) Distribución relacionada con la fracción gefúrica $(V) \mathrm{g} / \mathrm{f}$ cóncava y vacía, tipo plano y revestimientos de arcilla, con aumento de $20 x$, en PPL; B) Revestimientos de arcilla, con un aumento de 20x, en XPL (Fuente: Barbosa 2019).

aproximadamente el $10 \%$ corresponde a agujeros compuestos por cámaras; $10 \%$, del tipo complejo; y 5\%, plano (Fig. 11A), la distribución se considera aleatoria para el tipo de cámara. El ancho de los agujeros de la cámara es de entre $100 \mu \mathrm{m}$ y $300 \mu \mathrm{m}$. Con respecto a la matriz que compone la sección delgada, está compuesta en una relación g/f, que varía entre el $30 \%$ de la matriz gruesa y el $70 \%$ fina, con un color amarillo-naranja. La fracción fina es diferente en proporción y similar en composición a las dos matrices: color amarillo/naranja, básicamente compuesta de limo y arcilla, límpida y laminar, en PPL (Fig. 11A), con la presencia de pequeños puntos rojos, y la fábrica-b no está diferenciada, en XPL (Fig. 11B).

En la fracción gruesa están presentes granos de cuarzo con esfericidad sub-redonda, pobremente seleccionados, con distribución aleatoria (Fig. 12A), y fragmentos de nódulos de óxido de hierro anótico, con forma redondeada, surtidos, que representan el 1\% de la sección delgada y formación característica de los agregados. Es posible identificar la presencia de granos de cuarzo internos en el nódulo (Fig. 12B), con las mismas características que los granos de cuarzo presentes en la fracción gruesa. También se identificó un pequeño fragmento de cerámica en la fracción gruesa, en la cual es posible visualizar, en PPL (Fig. 12C), la textura de la arcilla, con la presencia de granos de cuarzo en la fracción fina a media, y en XPL (Fig. 12D), la presencia de antiplástico orgánico, ya que no tiene birrefringencia. En la fracción gruesa, las raíces también están presentes en forma fibrosa, con aproximadamente 5\%, y excrementos de ácaros elipsoides, siempre cerca de la presencia de las raíces. El carbón presente en $2 \%$ de la sección delgada tiene un contorno ondulado y pequeños revestimientos de arcilla en sus cavidades (Fig. 12E) y (Fig. 12F). Las características pedológicas de esta sección delgada incluyen nódulos y excrementos.

La recolecta CA-01 corresponde al horizonte A (antrópico), que se destaca no solo por la abundancia de fragmentos cerámicos identificados en la superficie, sino también por el color oscuro 10 YR 3/2 (seco). La porosidad total cubre aproximadamente el 40\% del área de la sección delgada. Alrededor del $20 \%$ corresponde a agujeros compuestos por cámaras; $5 \%$, por canales; y $15 \%$, por agujeros complejos, y estos varían entre $800 \mu \mathrm{m}$ y $200 \mu \mathrm{m}$. La rugosidad de los tipos de agujeros y canales de la cámara se considera aleatoria (Fig. 13B). La microestructura presente en la sección delgada del sitio Cangas I está entre el 60\% para la fracción gruesa y 40\% para la fracción fina, con distribución de espaciamiento simples (Single spaced fine enaulic) (Fig. 13A).

La fracción fina, como ya se mencionó, es diferente en proporción y similar en la composición de las dos matrices: color marrón oscuro a negro, básicamente compuesta de material orgánico, en PPL y XPL, y no presenta ninguna birrefringencia. Con respecto a la matriz de fracción mineral gruesa, los granos de cuarzo con esfericidad regular y pobremente seleccionada son dominantes, con la presencia de pequeńos fragmentos de petroplintita con esfericidad ondulada, relacionados 


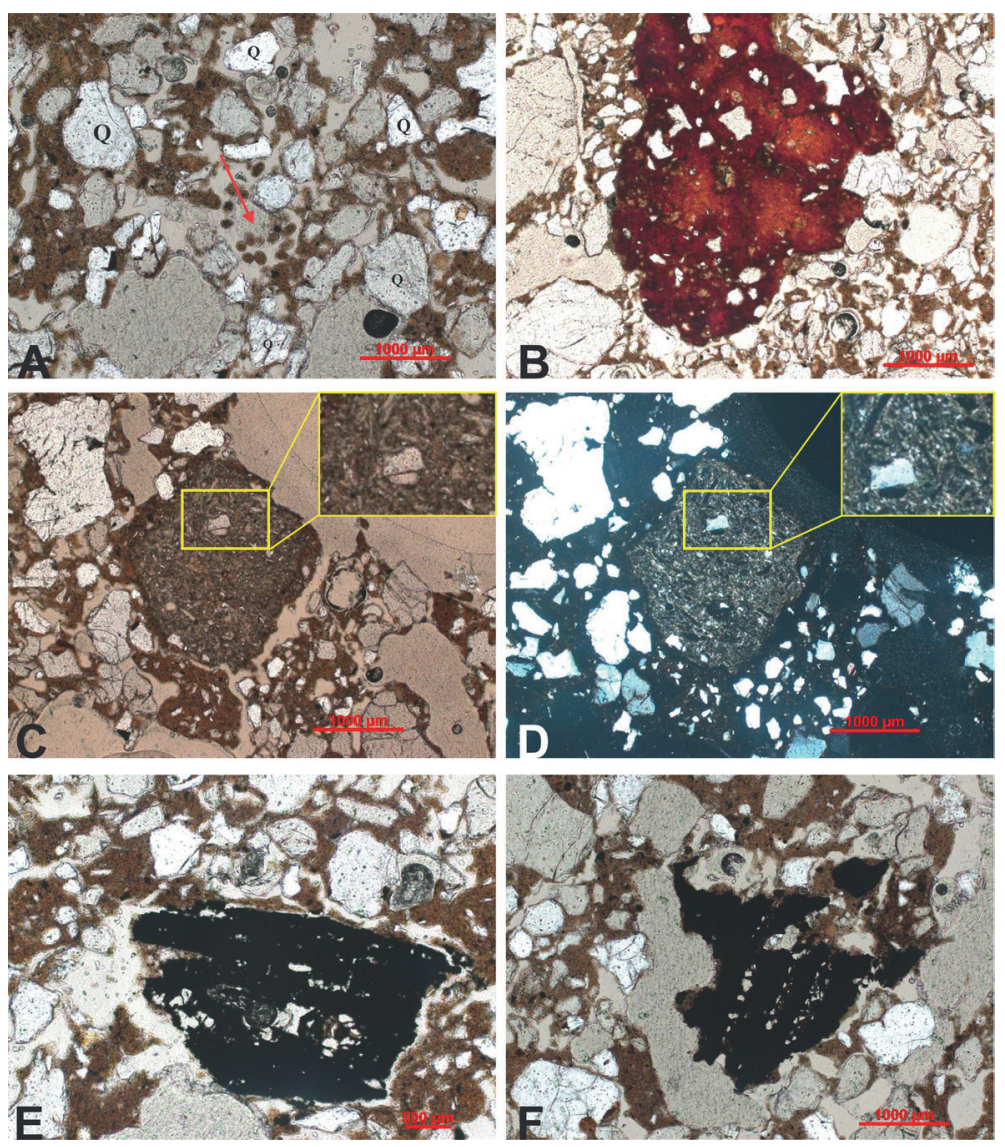

Figura 12. Fotomicrografias del portaobjetos LR-03: A) Cuarzo con esfericidad subangular y excrementos de elipsoide de ácaros, con aumento de 20x, en PPL; B) Nódulo anórtico, con un aumento de $5 x$, en PPL; C) Fragmento de cerámica, con 5 aumentos, en PPL; D) Fragmento de cerámica, con 5 aumentos, en XPL; E) Fragmento de carbón, con un aumento de 10x, en PPL; F) Fragmento de carbón, con un aumento de 10x, en PPL (fuente: Barbosa 2019).

con la geomorfogenia del paisaje o la formación del Araguaia. Hay presencia de fragmentos de cerámica arqueológica con forma globular y cilíndrica con distribución y orientación aleatorias (Figs. 13C y 13D).

En el fragmento cerámico es posible ver estructuras alargadas (Fig. 13E), posiblemente relacionadas con el antiplástico cauixi. En la figura $13 \mathrm{~F}$ podemos ver que estas estructuras están juntas con otra forma vegetal, con pequeños círculos. En la fracción orgánica gruesa, hay fragmentos de huesos milimétricos, con abundancia de aproximadamente el 8\%; fragmentos de carbón, presentes en 10\%; raíces, con 2\%; y excrementos, en un $2 \%$, estando estos dos últimos dentro de la fracción gruesa. Las variaciones de color identificadas en la sección delgada que pertenecen al sitio Cangas I varían de amarillo a rojo (Figs. 14A y 14B), de rojo a marrón (Fig. 14C y 14D) y, con mayor representación, fragmentos óseos incoloros (blanco) (Figs. 14E y 14F).

Los fragmentos de hueso siempre están unidos a fragmentos de carbón. Tienen una forma tabular (Fig. 15A y 15B) y se clasifican con distribución y orientación aleatorias. En esta fracción, también se ha identificado un tipo de tejido que aparentemente es escoria vesicular (Fig. 15C y 15D). Cuando los restos de tejido vegetal se queman, producen un tipo específico de residuo vítreo y no birrefringente con un tejido vesicular que puede presentarse en forma de agregados microcristalinos, formando estructuras como la escoria vesicular vitrificada criptocristalina (Courty et al. 1989). 

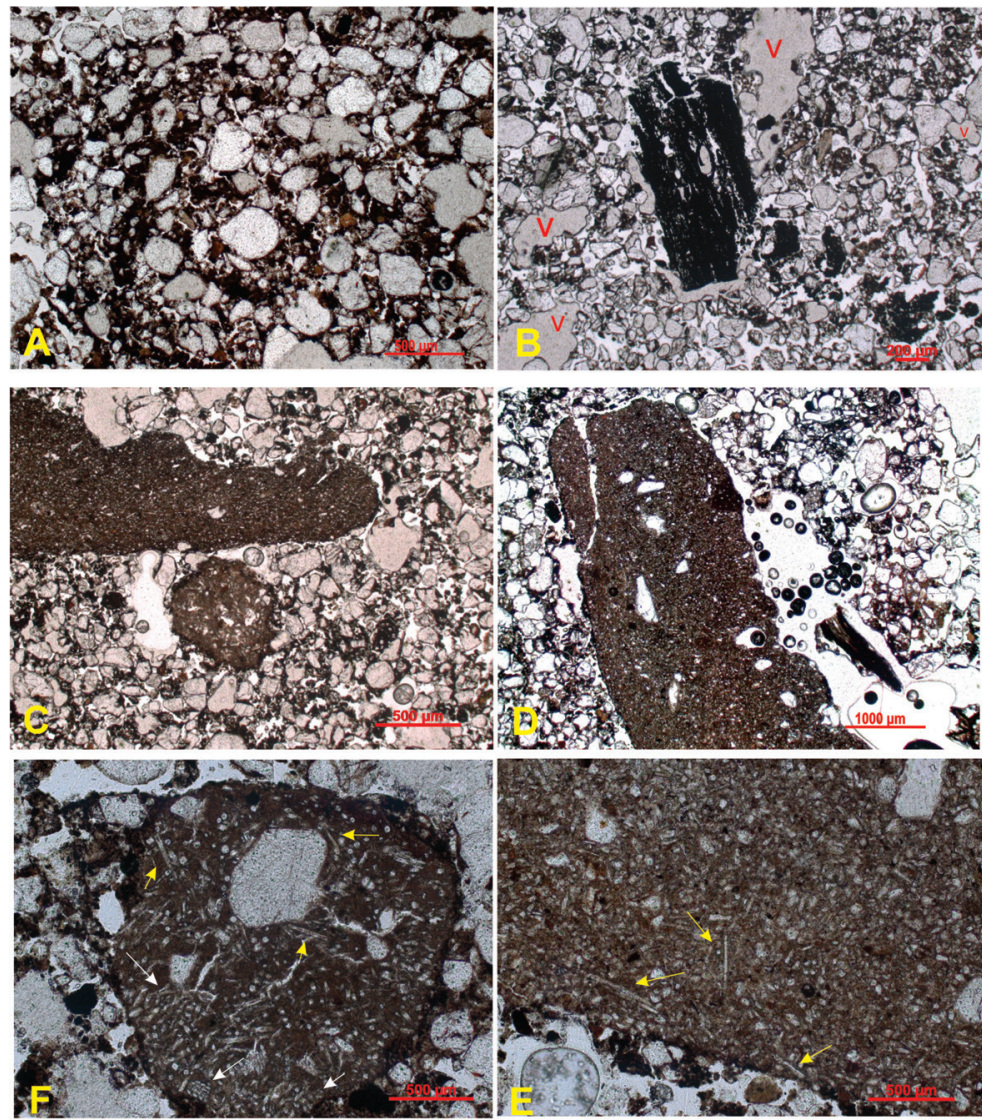

Figura 13. Fotomicrografias de la diapositiva CA 01: A) Distribución relacionada con la fracción $g / f$, aumento de 5x, en PPL; B) Tipo de cámara vacía (V), con fragmento de carbón con un aumento de $2.5 x$, en PPL; C) y D) Fragmentos de cerámica con 5 aumentos, en PPL; E) Fragmento cerámico con indicación de espigas cauixi; F) Pico de Cauixi, con aumento de 10x, en PPL; y fragmentos de tejidos orgánicos, 5x, en PPL (fuente: Barbosa 2019).

Aún en la fracción orgánica, se identificaron excrementos de ácaros elipsoides en las raíces, dentro o al lado de ellas. En la fracción gruesa, también hay poco excremento presente: en 1\%, con forma globular y distribución en grupos. Estos excrementos tienen una distribución y orientación aleatorias, se clasifican como mitoids, con coloración roja/amarilla y composición orgánica. Este tipo de excremento tiene una sección longitudinal alargada y, en la sección transversal, pequeños círculos.

\section{Discusión}

A lo largo de esta investigación determinamos que los suelos antrópicos alterados sin intención humana están compuestos, no solo por la presencia de objetos arqueológicos dentro de un suelo desarrollado, sino también por la presencia de matrices oscuras y micro trazas. En el laboratorio, se identificaron a escala microscópica procesos tafonómicos del suelo antrópicos, así como bioturbaciones, infiltración y percolación de arcilla.

Esta identificación se dio, en primer lugar, al acreditar que el sitio Lago Rico tiene un horizonte A antrópico que está presente a una profundidad de 30 centímetros y que, entre 30 y 40 centímetros, tenemos una transición del horizonte $\mathrm{A}$ al horizonte $\mathrm{Ab}$, con características mineralógicas de 

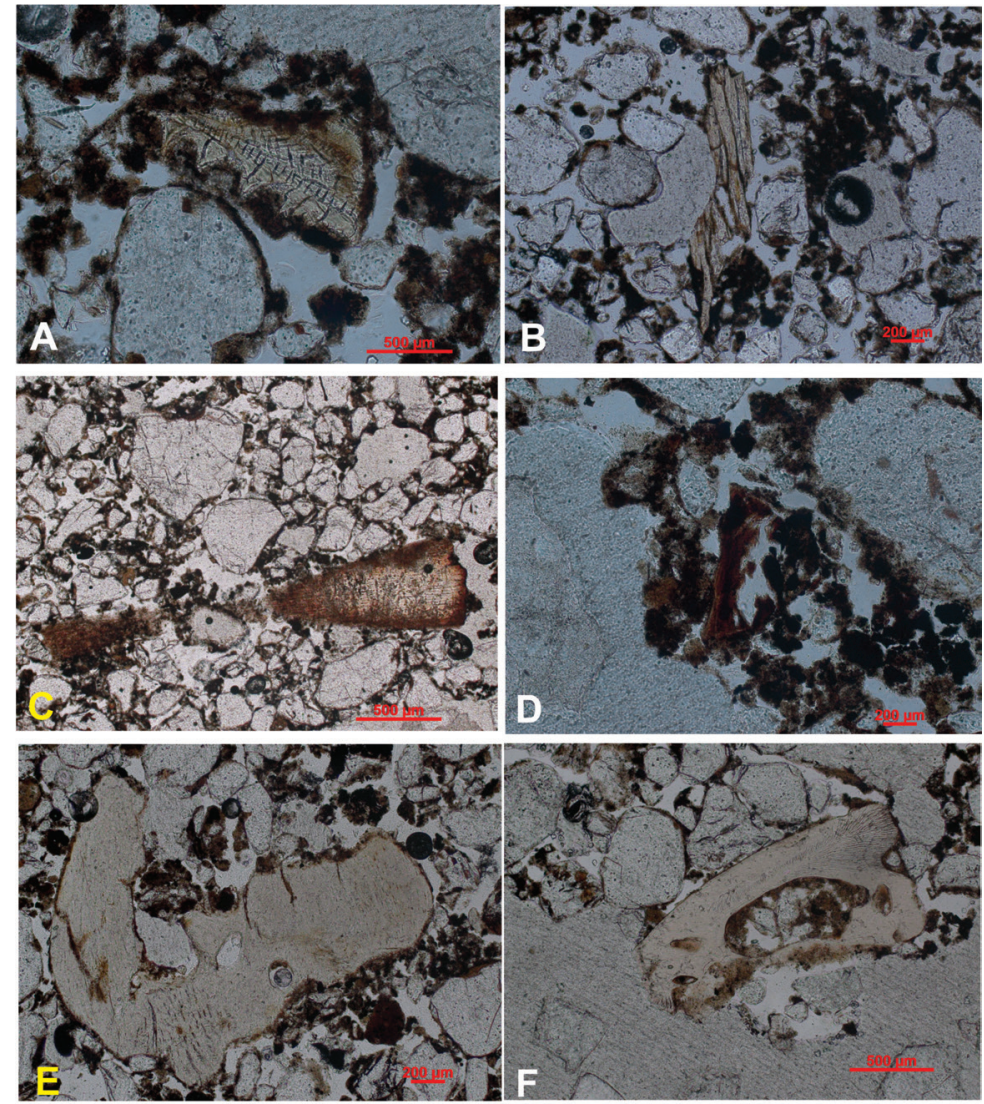

Figura 14. Fotomicrografías de la diapositiva CA 01: A) y B) Fragmentos óseos amarillos, con aumento de 20x, en PPL; C) Fragmento de huesos rojos / marrones, con un aumento de 5x, en PPL; D) Fragmento de huesos rojos / marrones, con 20 aumentos, en PPL; E) y F) Fragmentos óseos incoloros, con revestimiento de arcilla, aumento de 10x, en PPL (fuente: Barbosa 2019).

un horizonte natural. El Horizonte B no presenta rastros culturales a escala macro o micro, lo que muestra que los sitios de cerámica no son muy profundos.

Para este sitio, se identificó una matriz clara y fina con revestimientos de arcilla clara y laminar y una matriz gruesa, predominantemente de granos de cuarzo relacionados con la roca matriz, con restos de tejidos y fragmentos de carbón, nódulos y excrementos; además de la presencia de fragmentos cerámicos y formación de fosfatos secundarios en la fracción arcilla.

Para el sitio Cangas I, el horizonte antrópico A está presente hasta 40 centímetros de profundidad. El horizonte B aparece solo con 50 hasta 60 centímetros, lo que muestra una mayor intensidad de ocupación en el suelo. Con respecto a la composición, identificamos una fracción fina y oscura en la arcilla; y una fracción gruesa compuesta básicamente por granos de cuarzo y carbón. La fracción fina oscura puede estar relacionada con la cantidad de materia orgánica presente en el sitio. También hay fragmentos de cerámica, nódulos de arcilla, restos de tejidos orgánicos, así como microfragmentos óseos de diferentes colores y tamaños. También se identificó la presencia de un fragmento de escoria vesicular vitricular, material criptocristalino (sílice), que puede estar relacionado a un lugar de combustión y estabilidad al fuego.

Lo que indican los resultados son suelos extremadamente arenosos, con alrededor del 60\% al $70 \%$ de su composición formada a partir de meteorización en la roca matriz, presentando una textura que no excede el 15\% de arcilla, y una fracción de arena compuesta de granos de cuarzo. 


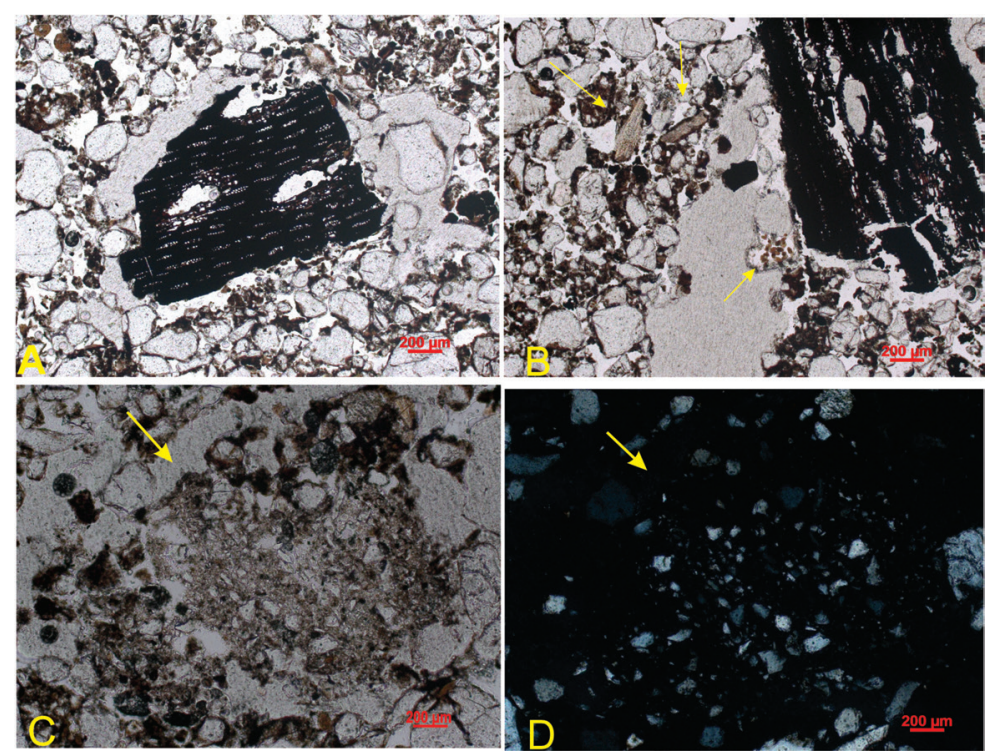

Figura 15. A) Fragmento de carbón, con un aumento de 5x, en PPL; B) Fragmento de carbón y fragmentos milimétricos de huesos y excrementos, con un aumento de $5 x$, en PPL; C) agregados de carbonato de calcio microcristalino, con un aumento de 10x, en PPL; D) Agregados microcristalinos de carbonato de calcio, con un aumento de 10x, en XPL (fuente: Barbosa 2019).

Los valores de $\mathrm{pH}$ encontrados para el sitio Cangas I varían de 4.2 hasta 6.2; en este rango, la acidez permite la conservación de otros materiales, como huesos y madera. Para el sitio Lago Rico, el rango de pH varía de 3.9 a 5.0 y se considera ácido moderado por la escala de pH (Santos et al 2018).

Teniendo en cuenta estos resultados de textura y $\mathrm{pH}$ para los sitios arqueológicos Cangas I y Lago Rico, lo que los diferencia es la presencia de un $\mathrm{pH}$ más moderado para el sitio Cangas I. El pH encontrado en el sitio Cangas I permitió la prolongación de valores de $16 \mathrm{~g} / \mathrm{dm} 3$, alcanzando hasta $20 \mathrm{~g} / \mathrm{dm} 3$, de materia orgánica y valores de 6 a $11 \mathrm{~g} / \mathrm{dm} 3$ de carbono, así como valores de $\mathrm{P}$ superiores a $30 \mathrm{ppm}$ también asociados con los valores $\mathrm{Zn}, \mathrm{Ca}, \mathrm{Mn}$ y K. Estos valores son similares a la identificación de áreas de relleno sanitario en aldeas precoloniales en el Alto Xingu (Heckenberger 2005; Schmidt y Heckenberger 2009; Schmidt 2010). Estas cifras no se repiten para el sitio de Lago Rico. Los bajos valores encontrados pueden estar asociados con lo que Woods (2002) menciona sobre la pérdida de nutrientes por el proceso de lixiviación, o estar presentes en un porcentaje más bajo que los otros elementos, lo cual se demostró en el horizonte B del sitio. En suelos del bioma Cerrado, el contenido de materia orgánica es bajo, generalmente entre 3\% y 5\% (Coutinho 2016).

A partir de los resultados, observamos que los mismos elementos presentes en el sitio Lago Rico están presentes en el sitio Cangas I, siendo la diferencia en relación a la cantidad de estos componentes. La variación en los resultados muestra que en el sitio Cangas I, la vegetación local puede haber permitido la estabilización de carbono y materia orgánica en el suelo, y la actividad microbiana, además de mantener la humedad, reduciendo la lixiviación de nutrientes para el horizonte $\mathrm{B}$, manteniendo la fertilidad del suelo, además de las firmas químicas, en el horizonte antrópico, desde un punto de vista tafonómico, bien conservadas.

Los resultados obtenidos en la lectura de las secciones delgadas exponen que el origen de la matriz ennegrecida, es el resultado de una mayor cantidad de residuos orgánicos, como el carbón vegetal y los tejidos vegetales. 
Teniendo en cuenta las hipótesis de Marcos en 2011, de que el sitio Cangas I podría ser un sitio temporal, mientras que el Lago Rico sería una aldea principal. Esta hipótesis no se comprueba, ya que el sitio Lago Rico no presentó, estructura de fogata, viviendas, áreas de descarte o incluso fragmentos de carbón; mientras que el sitio Cangas I presentaba estructuras de fogatas, marcas de pilares, fragmentos de carbón fechados, fragmentos de hueso macro y micro identificados en el campo y en el laboratorio; así como la prueba del origen antrópico de las firmas químicas encontradas en los sitios arqueológicos debido a actividades humanas precoloniales.

De esta manera, los resultados obtenidos de los métodos de la geoquímica y micromorfología demuestran que el sitio Cangas I es el sitio arqueológico más antiguo con características de ocupación fija identificadas por depósitos antrópicos y fechas más antiguas, mientras que el sitio de Lago Rico estaría ocupado al mismo tiempo como un campamento de movilidad estacional.

\section{Comentarios finales}

A lo largo de más de cinco décadas, el centro de Brasil ha sido objeto de investigaciones relacionadas a grupos alfareros en los estados de Goiás, Mato Grosso y Mato Grosso do Sul, cuyo objetivo ha sido tratar de comprender las relaciones que los grupos alfareros mantenían entre ellos (Schmitz et al. 1982; Wüst 1983, 1990; Wüst y Barreto1999; Robrahn-González 1996; Wüst y Barreto 1999; Heckenberger 2001; Schmidt 2010). Las grandes aldeas alfareras que se encuentran en el centro de Brasil son asentamientos al aire libre, poco estratificados, pero son espacios físicos donde vivían grupos de personas presentes allí desde al menos el siglo IX. Por lo tanto, el uso de nuevas perspectivas, dirigidas al estudio de estas ocupaciones alfareras, puede ampliar la comprensión de cómo vivieron estas poblaciones en los últimos dos mil años.

\section{Agradecimientos}

Los autores desean agradecer a la Fundação de Amparo à Pesquisa do Estado de Sáo Paulo (FAPESP, proceso 201 /22192-7) y la Beca de Investigación de Pasantías en el Extranjero - BEPE (proceso: 2018/01377- 4) y la Coordenação de Aperfeiçoamento Pessoal de Nível Superior (CAPES), por la beca de maestría en la realización de esta investigación, entre 2017 y 2019. Agradecen al laboratorio de suelos (EMBRAPA-RJ); al laboratorio de Geoarqueología de la Universidad de Tübingen y al laboratorio de Microarqueologia de la Universidad de São Paulo - MAE. Agradecemos por las becas CNPq - Brasil: Marisa Coutinho Afonso, Julio Cezar Rubin de Rubin (procesos números 310373/2016-6 e 307188/2019-1).

\section{Notas}

1. La fase de Mossâmedes fue definida por Wüst (1983) en los años 1975 y 1976, delimitada en el suroeste del estado de Goiás. Instalados al aire libre con las características de las grandes aldeas de la tradición Aratu con grandes contenedores piriformes.

2. Se trata de diferentes especies de árboles que se queman y se usan como especias en la pasta cerámica, cuyas cortezas son ricas en sílice (Viana et al. 2011).

3. Corresponde al período terciario que consiste en sedimentos continentales no consolidados, con unidades de conglomerado, limos y arenas consolidadas, de color amarillo a marrón rojizo y con granulometría variable (Bayer 2002, 2010).

4. Corresponde a la matriz que conforma la sección delgada, con dominios de distribución aleatoria, el cociente en relación con la diferencia entre grueso/fino $(\mathrm{g} / \mathrm{f})$ es el volumen que ocupan en la paleta las dos fracciones (Stoops 2003). 


\section{Referencias}

Andreatta, M.D. (1975). Projeto Anhanguera de Arqueologia de Goiás, Revista do Museu Paulista N S. 1988-33, 143-156.

Andreatta, M.D. (1982). Padrōes de povoamento em Pré-História goiana: análise de sítio tipo, tesis de doctorado [Doutorado em Antropologia], Faculdade de Filosofia, Letras e Ciências Humanas /Universidade de São Paulo, FFLCH/USP.

Araujo, A. G. M., J.K Feathers, M. Arroyo-Kalin y M.M Tizuka (2008). Lapa das Boleiras rockshelter: stratigraphy and formation processes at a Paleoamerican site in Central Brazil, Journal of Archaeological Science 35(12), 3186-3202. https://doi.org/10.1016/j.jas.2008.07.007

Arroyo-Kalin, M. (2008). Steps towards an ecology of landscape: a geoarchaeological approach to the study of anthropogenic dark earths in the central Amazon region, Brazil, tesis de doctorado, Department of Archaeology, University of Cambridge, Cambridge.

Arroyo-Kalin, M. (2014). Anthropogenic sediments and soils, Geoarchaeology, en: C. Smith (ed.), Encyclopedia of Global Archaeology, 279-284, New York.

Bahia Filho, A.F., J.M. Braga y M. Resende (1983). Relação entre absorção de fósforo e componentes mineralógicos da fração argila de Latossolos do Planalto Central. R. Bras. Ci. Solo Brasília, 221-226.

Barbosa, J.B. (2015). Tempo, marcas e vestígios na paisagem vivida: arqueoestratigrafia do sítio Lago Rico - Aruanã Goiás, monografía [Graduação em Arqueologia], Pontifícia Universidade Católica de Goiás, Goiânia.

Barbosa, J.B. (2019). A aplicação da geoquímica e micromorfologia na detecção de solos antrópicos em assentamentos ceramista Uru na bacia do rio Araguaia, Goiás, Brasil, tesis de maestría [Dissertação Mestrado], Museu de Arqueologia e Etnologia da Universidade de São Paulo, MAE/USP.

Barreto, M.V. (2010). Abordando o passado: uma introdução à Arqueologia, Paka-Tatu Ltda, Bélem.

Bayer, M. (2002). Diagnostico dos processos de erosao/assoreamento na planicie aluvial do rio Araguaia: entre Barra do Garcas e Cocalinho, tesis de maestría [Dissertação de Mestrado], Universidade Federal de Goias, Goiânia.

Bayer, M. (2010). Dinâmica do transporte, composição e estratigrafia dos sedimentos da planicie aluvial do rio Araguaia, tesis de doctorado, Universidade Federal de Goiás, Goiânia.

Bennema, J. (2006[1966]). Classification of Brazilian soils, reporte al Gobierno de Brasil. Roma: FAO.

Bullock, P., N. Fedoroff, A. Jongerius, G. Stoops, T. Tursina y U. Babel (1985). Handbook for soil thin section description, Waine Research Publications, Wolverhampton.

Correa, D.S. (2013). Pedologia e Subsistência: Sítio Arqueologico Pré-Colonial Lago Rico, Aruanã - Goiás, monografia [Graduação em Arqueologia], Pontificia Universidade Católica de Goiás, Goiânia.

Costa, J.A., D.C. Kern, C.M. Lima, T.E. Rodrigues, N. Kämpf, J. Lehmann y F.J.L. Frazão (2009). Geoquímica das Terras Pretas Amazônicas, en: W.G. Teixeira, D.C. Kern, B.E. Madari, H.N. Lima y W. Woods (eds.), As terras pretas de indio da Amazônia: sua caracterização e uso deste conhecimento na criação de novas áreas, Manaus: Embrapa Amazônia Ocidental, 172:171.

Courty, M. A., P. Goldberg y R. Macphail (1989). Soils and micromorphology in archaeology, Cambridge University Press, Cambridge.

Coutinho, L.M. (2016). Biomas brasileiros, Oficina de Textos, São Paulo.

Denevan W.M. (2004). Semi-intensive pre-European cultivation and the origins of anthropogenic Dark Earths in Amazonia, en: B. Glaser y W.I. Woods (eds.), Amazonian Dark Earths: Explorations in space and time, 135-143, Springer, Berlín.

Dubois, F. (2009). Geoarqueología: explorando propiedades espaciales y temporales del registro arqueológico, en: R. Barbarena. K. Borrazzo y L.A. Borrero (eds.), Perspectivas actuales en la arqueología argentina, 35-54, CONICET-Imhicihu, Buenos Aires.

Erickson, C. (2003). Historical ecology and future explorations, en: J. Lehmann, D.C. Kern, B. Glaser, W.I. Woods (eds.), Amazonian dark earths: origin, properties, management, 455-500, Kluwer, Dordrecht.

Eidt, R.C. y W. Woods (1974). Abandoned settlement analysis: Theory and practice, Field Test Associates, Wisconsin.

Eidt, R.C. (1985). Theoretical and practical considerations in the analysis of anthrosols, en: G. Rapp y J. A. Gifford (eds.), Archaeological Geology, Yale University Press, New Haven/London.

Estrela, V.P. (2017). Análise Cerâmica da Unidade de Escavação 3 do Sítio Lago Rico (Aruanā/Goiás), monografía [Graduação em Arqueologia] , Pontificia Universidade Católica de Goiás, Goiânia.

Feathers, J., R. Kipnis, L. Piló, M. Arroyo-Kalin y D. Coblentz (2010). How old is Luzia? Luminescence dating and stratigraphic integrity at Lapa Vermelha, Lagoa Santa, Brazil, Geoarchaeology 25(4), 395-436. 
Glaser, B. (2007). Prehistorically modified soils of central Amazonia: A model for sustainable agriculture in the twenty-first century, Phil. Trans. R. Soc. B.p. 362(1478), 187-196. https://doi.org/10.1098/rstb.2006.197

Goldberg, P. y R. McPhail (2006). Practical and Theoretical Geoarchaeology, Blackwell Publishing, Oxford.

Hecht, S. (2003). Indigenous soil management and creation of Amazonian dark earths: implications of kayapó practices, en: J. Lehmann, D. Kern, B. Glaser y W.I. Woods, Amazonian Dark Earths: origin, properties, management, 355-372, Kluwer Academic Publishers, Dordrecht.

Heckenberger, M.J. (2001). Estrutura, história e transformação: a cultura Xinguano no longue duree, en: B. Franchetto y M.J. Heckenberger (eds.), Os Povos do Alto Xingu: História e Cultura, 21-62, Editora da Universidade Federal do Rio de Janeiro, Rio de Janeiro.

Heckenberger, M.J. (2005). The ecology of power: culture, place, and personhood in the southern Amazon, AD 10002000. Routledge, New York. https://doi.org/10.2307/971962

Heckenberger, M.J., J.B. Petersen y E.G. Neves (1999). Village size and permanence in Amazonia: Two archaeological examples from Brazil, Latin American Antiquity 10(4), 353-376.

Holliday V.T. (2004). Soils in archaeological research, Oxford University Press, Oxford.

Howard, J. (2017). Anthropogenic Soils, Springer International Publishing.

IDESP (1974). Recursos de solos estudos integrados da Ilha de Marajó, 236-292, Belém.

IUSS Grupo de Trabajo WRB (2007). Base Referencial Mundial del Recurso Suelo. Primera actualización 2007. Informes sobre Recursos Mundiales de Suelos No. 103. Roma: FAO. Recuperado de http://www.fao.org/3/ a0510s/a0510s00.htm

Kern, D.C., M.L. Costa y M.L.P. Ruivo (2009). Métodos e técnicas geoarqueológicas para caracterizaçáo de solos com Terra Preta na Amazônia: contribuiçóes para a Arqueologia, en: J.C.R. Rubin, R.T. Silva (eds.), Geoarqueologia: teoria e prática 7, 133-152, Ed. da UCG, Goiânia.

Lahaye, C., G. Guérin, M. Gluchy, C. Hatté, M. Fortugne, Clemente-Conte, J.C. Santos, X.S. Villagrán, A. da Costa, C. Borges, N. Guidon y E. Boeda (2019). Another site, same old song: The Pleistocene-Holocene archaeological sequence of Toca da Janela da Barra do Antoniāo-North, Piauí, Brazil, Quaternary Geochronology 49, 223-229. https://doi.org/10.1016/j.quageo.2018.03.006

Marcos, S.D. (2011). Área de captação de recursos do sítio arqueológico Cangas I, Aruanä, Relatório de Iniciaçáo Científica, Pontifícia Universidade Católica de Goiás, Goiânia.

Macedo, R.S., W.G. Teixeira, H.N. Lima, A.C.G. Souza, F.W.R. Silva, O.C. Encinas y E.G. Neves (2019). Amazonian dark earths in the fertile floodplains of the Amazon River, Brazil: example of non-intentional formation of anthropic soils in the Central Amazon region, Boletim do Museu Paraense Emilio Goeldi. Ciências Humanas 14, 207-227. https://doi.org/10.1590/1981-81222019000100013

Macphail, R. y J. Cruise (2001). The soil micromorphologist as team player: a multianalytical approach to the study of European microstratigraphy, en: R. Goldberg, V.T. Holliday y C.R. Ferring (eds.), Earth Sciences and Archaeology, 241-267, Kluwer Academic/Plenum Publishers, New York.

Mello, F.A.F., M.O.C. Brasil Sobrinho, S. Arzolla, R.I. Silveira, A. Cobra Netto y J.C. Kiehl (1983). Fertilidade do solo, Nobel, São Paulo.

Melo, J. y M.A. Telles ( 2009). Projeto de levantamento e Resgate arqueológico na área de influência direta da fazenda dois Rios, informe, Lagoa das Confusóes, Tocantins.

Morais, J.L., R.L. F. Bucci y E.J.F. Reis (1998). Subprograma Geoarqueologia, en: D.C. Martins (coord.), Relatório Conclusivo do Programa Arqueologico-Salvamento-Serra da Mesa, UFG, informe, Universidade Federal do Estado de Goiás/Museu Antropológico 3, Goiânia.

Moreira, M.L.O., L.C. Moreton, V.A. Araujo, J.V. Lacerda-Filho y H.F. Costa (2008). Geologia do Estado de Goiás e Distrito Federal, CPRM/SIC-FUNMINERAL, Goiânia.

Moura, E.S. (2015). Geoarqueologia fluvial do Sítio Lago Rico, monografia [Graduação em Arqueologia], Pontifícia Universidade Católica de Goiás, Goiânia.

Oliveira, J.E., Viana, S.A. (1999-2000). O centro-oeste antes de Cabral. São Paulo, Revista do Museu Paulista 44, 142-189. https://doi.org/10.11606/issn.2316-9036.v0i44p142-189

Ortega, D.D. (2016). A cerâmica arqueológica do sítio Lago Rico: questôes sobre funcionalidade, funcionamento e função, monografia [Graduação em Arqueologia], Pontifícia Universidade Católica de Goiás, Goiânia.

Pereira, T.M. (2010). Complementaçâo da análise da cerâmica da sondagem S2 do Sitio Cangas I, Aruanã-Goiás, Relatório final de iniciação científica, Pontifícia Universidade Católica de Goiás, Goiânia.

Posey, D.A (1984). Preliminary Report on Diversified Management of Tropical Forest by the Kayapo Indians of the Brazilian Amazon, Advances in Economic Botany 1, 112-126.

Robrahn-González, E. (1996). Os grupos ceramistas pré-coloniais do Centro-Oeste brasileiro, Revista do Museu de Arqueologia e Etnologia 6, 83-121. 
Rosa, O.B. (2009). Análise dos processos formadores do registro arqueológico da sondagem 1 do sítio arqueológico Cangas 1, Aruanã, Goiás, monografia [Graduação em Arqueologia], Pontifícia Universidade Católica de Goiás, Goiânia.

Rubin, J.C. (2007). Abordagem Geoarqueológica na Prospecção do Sitio Cangas I, terraço aluvial do rio Araguaia, Goiás, Pontifícia Universidade Católica de Goiás, Goiânia.

Rubin, J.C.R., R.T. Silva, M. Bayer, M. Barberi, J.B. Barbosa, D.D. Ortega, V.P. Estrela, J.E. Ribeiro-Freitas y S.A. Viana (2019). Ocupaçáo pré-colonial na bacia do rio Araguaia, Brasil, en: M. Bonomo y J.C.R. Rubin (eds.), Arqueologia e Rios na América do Sul, Revista del Museo de la Plata 4(2), 401-436.

Ruivo, M.L.P., M.A. Arroyo-Kalin, C.E.R. Schaefer, H.T. Costi, S. H. de Souza Arcanjo, H.N. Lima, M.M. Pulleman y D. Creutzberg (2004). The use of micromorphology for the study of the formation and properties of Amazonian dark earths, en: J. Lehmann, D.C. Kern, B. Glaser y W.I. Woods (eds.), Amazonian dark earths: origin, properties, management, 243-254. Kluwer Academic Publishers, Boston.

Santos, H.G., P.K.T. Jacomine, L.H.C. Anjos, V.A. Oliveira, J.F. Lumbreras, M.R. Coelho, J.A. Almeida, J.C. Araújo Filho, J.B. Oliveira y T.J.F. Cunha (2018). Sistema Brasileiro de Classificaçâo de Solos, $5^{\text {ta }}$ ed., Embrapa, Brasília.

Schmidt, M. J. (2010). Reconstructing tropical nature: Prehistoric and modern anthrosols (Terra Preta) in the Amazon Rainforest, Upper Xingu River, Brazil, tesis de doctorado, University of Florida.

Simonsen, I. (1983-1984 Sítios cerâmicos da bacia do Paranã-Goiás, Arquivos do Museu de História Natural de Belo Horizonte 8- 9, 121-129.

Schiffer, M.B. (1972). Archaeological context and systemic context, American Antiquity 37(2),156-165. https:// doi.org/10.2307/278203

Schmitz, P. I. (1986). Caiapônia, IAP-Unisinos, São Leopoldo.

Schmitz, P.I., A.S. Barbosa e I. Wüst (1976). Arqueologia de Goiás 1976, informe, Instituto Anchietano de Pesquisas, Universidade do Vale do Rio dos Sinos, Goiânia.

Schmitz, P.I. y A.S. Barbosa (1985). Horticultores pré-históricos do Estado de Goiás, IAP-Unisinos São Leopoldo.

Schmitz, P.I., I. Wüst y S.M. Copé (1982). Os horticultores do centro-sul de Goiás, informe, Arquivos do Museu de História Natural da UFMG, 221-234, Belo Horizonte.

Schmidt, M.J. y M.J. Heckenberger (2009). Formação de Terra Preta na Região do Alto Xingu: Resultados Preliminares, en: W.G.Teixeira, D. Kern, B.E. Madari y H. Lima (eds.), As terras pretas de indio da Amazônia: sua caracterização e uso deste conhecimento na criação de novas áreas, 103-126, Embrapa Amazônia Ocidental, Manaus.

Silva, F.A. y L. Rebellato (2004). Use of space and Terra Preta formation: The Asurini do Xingu case study, en: B. Glaser y W.I. Woods (eds.), Amazonian dark earth: Exloration in space and time, 159-167, Springer, Berlin.

Silva, S.M. (2013). Área de Captação de Recursos do sitio arqueológico Cangas I, Aruanã - Goiás, novas abordagens e novas perspectivas, monografia [Graduação em Arqueologia], Pontifícia Universidade Católica de Goiás, Goiâna.

Sirico, L.H.A. (2010). Os Aldeamentos De Agricultores Ceramistas: O Caso do GO-Ja.33 Sítio Jaguarundi, sudoeste Goiano, dissertaçấo [Mestrado em Arqueologia], Museu de Arqueologia e Etnologia da Universidade de São Paulo, São Paulo.

Sousa, D.M.G., E. Lobato y T.A. Rein (2002). A adubaçấo com fósforo, en: D.M.G. Sousa y E. Lobato (eds.), Cerrados: correção do solo e adubação, 147-168, Embrapa Cerrados, Planaltina.

Sousa, D.M.G. y E. Lobato (2004). Adubação fosfatada em solos da região do Cerado, en: T. Yamada. S.R. Stipp y Abadalla. (eds.), Fósforo na agricultura brasileira, 157-196, Instituto da Potassa e Fosfato, Piracicaba.

Souza, M.L. (2003). Estudos de sítios pré-coloniais na bacia do rio Tocantins: análise arqueológica, [tesis de doctorado em Arqueologia], Museu de Arqueologia e Etnologia da Universidade de São Paulo, São Paulo.

Stoops, G. (2003). Guidelines for analysis and description of soil and regolith thin sections, Soil Science Society of America, Wiley.

Teixeira, W.G. y R.L. Lima (2016). O solo modificado pelo homem (solo antrópico) como artefato arqueológico, Anais do Seminário de Preservação de Patrimônio Arqueológico 4, Museu de Astronomia e Ciências Afins, Rio de Janeiro.

Viana, S.A., C.V. Ribeiro y S.D. Oliveira (2011). Cauixi em cerâmica arqueológica: uma questão de escolhas culturais, Revista de arqueologia, São Paulo 24(1), 32-51.

Villagran, X.S. (2008). Análise de arqueofácies na camada preta do sambaqui Jabuticabeira II, dissertaçáo [Mestrado em Arqueologia], Museu de Arqueologia e Etnologia da Universidade de São Paulo, São Paulo. 
Villagran, X.S. (2014). Experimental micromorphology on burnt shells of Anomalocardia brasiliana (Gmelin 1791) (Bivalvia, Veneridae) and its potential for identification of combustion features on shell-matrix sites, Geoarchaeology 29, 389-396. https://doi.org/10.1002/gea.21486

Villagran, X.S. (2019). The shell midden conundrum: Comparative micromorphology of shell-matrix sites from South America, Journal of Archaeological Method and Theory 26, 344-395. https://doi.org/10.1007/s10816018-9374-2

Villagran, X.S., P.C.F. Giannini, y P. DeBlasis (2009). Archaeofacies analysis: using depositional attributes to identify anthropic processes of deposition in a monumental shell mound of Santa Catarina State, southern Brazil, Geoarchaeology 24, 311-335. https://doi.org/10.1002/gea.20269

Villagran, X.S., D. Klokler, P. Nishida, M.D. Gaspar y P. DeBlasis (2010). Lecturas estratigraficas: arquitetura funeraria y depositación de residuos en el sambaquí Jabuticabeira II, Latin American Antiquity 21, 195-216.

Villagran, X., D. Klokler, S. Peixoto, P. Deblasis y P. Giannini (2011). Building coastal landscapes: zooarchaeology and geoarchaeology of Brazilian shell mounds, Journal of Island and Coastal Archaeology 2, 211-234.

Villagran, X.S. y P.C. Giannini (2014). Shell mounds as environmental proxies on the southern coast of Brazil, The Holocene 24, 1009-1016. https://doi.org/10.1177/0959683614534743

Villagran, X.S., Strauss, A., Miller, C., Ligouis, B., Oliveira, R. (2017). Buried in ashes: Site formation processes at Lapa do Santo rockshelter, east-central Brazil, Journal of Archaeological Science 77, 10-34. https://doi. org/10.1016/j.jas.2016.07.008

Woods, W.I. (2002). Perspectives on Terra Preta Soils, ponencia presentada en Perspectives and Reflections on the Anthropogenic Amazon panel 98th annual Meeting of the association of American Geographers, Los Angeles.

Wüst, I., Barreto, C. (1999). The ring villages of central Brazil: a challenge for Amazonian archaeology, Latin American Antiquity 10(1), 3-23.

Wüst, I. (1983). Aspectos da Ocupação Pré-Colonial em uma Área do Mato Grosso Goiás - Tentativa de Análise Espacial, dissertação [Mestrado em Arqueologia], Universidade de Sáo Paulo, São Paulo.

Wüst, I. (1990). Continuidade e mudança: para uma interpretação dos grupos pré-coloniais na Bacia do rio Vermelho, Mato Grosso, tesis de doctorado [Doutorado em Antropologia Social], Faculdade de Filosofia, Letras e Ciências Humanas da Universidade de São Paulo, São Paulo.

Zeidler, J.A. (1983). La etnoarqueología de una vivienda achuar y sus implicaciones arqueológicas, Boletín de los Museos del Banco Central del Ecuador 3, 156-194; Quito: Miscelánea Antropológica Ecuatoriana.

\section{Páginas web}

IBGE - Instituto Brasileiro de Geografia e Estatística (2020). https://www.ibge.gov.br/ SIEG - Sistema Estadual de Geoinformação (2017). https://www.imb.go.gov.br/sieg

Recibido: 06/07/2020

Aprobado: 8/10/2020 